\title{
De la Renaissance à la Restauration : quelques étapes du déchiffrement des hiéroglyphes
}

From the Renaissance to the Bourbon Restoration: milestones in the decipherment of (Egyptian) hieroglyphs

\section{Dominique Farout}

\section{CpenEdition}

Journals

Édition électronique

URL : http://journals.openedition.org/cel/433

DOI : $10.4000 / \mathrm{cel} .433$

ISSN : 2262-208X

Éditeur

École du Louvre

Référence électronique

Dominique Farout, « De la Renaissance à la Restauration : quelques étapes du déchiffrement des hiéroglyphes », Les Cahiers de l'École du Louvre [En ligne], 9 | 2016, mis en ligne le 15 décembre 2016, consulté le 17 septembre 2019. URL : http://journals.openedition.org/cel/433; DOI : 10.4000/cel.433

Ce document a été généré automatiquement le 17 septembre 2019.

\section{cc) (1) $\odot$}

Les Cahiers de l'École du Louvre sont mis à disposition selon les termes de la licence Creative Commons Attribution - Pas d'Utilisation Commerciale - Pas de Modification 4.0 International. 


\section{De la Renaissance à la Restauration : quelques étapes du déchiffrement des hiéroglyphes}

From the Renaissance to the Bourbon Restoration: milestones in the

decipherment of (Egyptian) hieroglyphs

Dominique Farout

\section{Les prémices : la Renaissance, de Blaise de Vigenère à Athanasius Kircher}

1 Chercher un monument bilingue pour faire renaître une écriture disparue depuis des siècles n'est pas naturel. L'aventure du déchiffrement est un phénomène unique dans l'histoire de l'humanité, qui résulte d'un enchaînement d'évènements accidentels. Pour en comprendre les raisons et les modalités, il faut remonter à la Renaissance. En effet, l'intérêt pour les hiéroglyphes d'une part et pour le déchiffrement d'autre part sont des « dommages collatéraux » de cette révolution intellectuelle sans précédent.

\section{Des obélisques}

2 Le goût pour l'Antiquité est une caractéristique de la Renaissance, comme l'indique le nom de cette période. Pour retrouver l'Antique, on fait des fouilles à Rome qui dévoilent des monuments égyptiens, en particuliers des obélisques couverts de hiéroglyphes. Pour la même raison, on retrouve des écrits anciens traitant, d'une manière ou d'une autre, de l'écriture pharaonique.

3 À Rome, dès 1373, on met au jour l'obélisque du Panthéon inscrit aux noms de Ramsès II. Au cours du $\mathrm{xVI}^{\mathrm{e}}$ siècle, les découvertes de monuments couverts de hiéroglyphes se succèdent. On sort de terre la Mensa Isiaca. On transporte l'obélisque de la Villa Celimontana, livrant la titulature de Ramsès II. On retrouve l'obélisque du 
Pincio qui porte un texte hiéroglyphique relatif à Hadrien et Antinoos, l'obélisque du Latran aux noms de Thoutmosis III, les obélisques de la Trinité des Monts et de la Piazza del Popolo, aux noms de Séthi I ${ }^{\text {er }}$ et Ramsès II, ainsi que l'obélisque du Montecitorio qui porte la titulature de Psammétique II. Au XvII ${ }^{e}$ siècle, on érige l'obélisque de la piazza della Minerva aux noms d'Apriès - celui de l'éléphant du Bernin (fig. 1) - et l'obélisque de la piazza Navona qui porte des hiéroglyphes aux noms de l'empereur Domitien.

Fig. 1

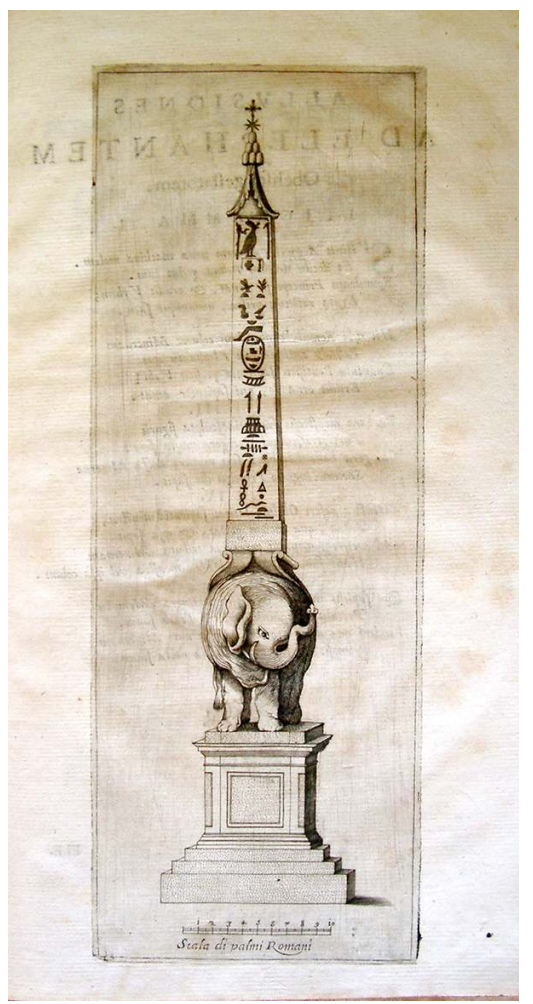

Gravure de l'obélisque de la place Santa Maria della Minerva à Rome - D'après Athanasius Kircher, Obelisci Aegyptiaci nuper inter isaei romani rudera effossi interpretatio hieroglyphica, Rome, Varesi, 1666 Bibliothèque universitaire d'Heidelberg, C 2901 FOL

En 1414, on découvre, dans un monastère allemand, la traduction grecque attribuée par Ammien Marcellin ${ }^{1}$ à un certain Hermapion ${ }^{2}$ des inscriptions hiéroglyphiques couvrant la surface d'un obélisque d'Héliopolis déplacé à Rome par l'empereur Auguste. (17) Quant à l'ancien obélisque, celui du grand cirque, Hermapion en a traduit en grec les inscriptions emblématiques, et voici son interprétation. D'abord la face du sud.

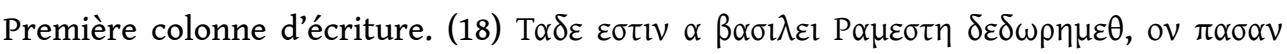

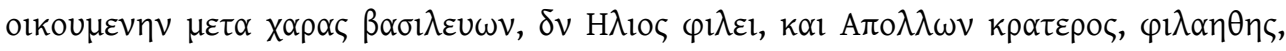

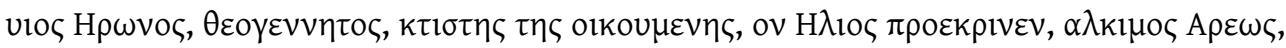
$\beta \alpha \sigma \lambda \lambda \varepsilon \cup \varsigma$ P $\alpha \mu \varepsilon \sigma \tau 1 \zeta, \omega \pi \alpha \sigma \alpha \eta \eta \pi \tau \varepsilon \tau \alpha \kappa \tau \alpha l \eta \eta \eta \eta$ $\mathrm{H} \lambda \lambda_{10} \pi \alpha ı \varsigma, \alpha 1 \omega v o \beta ı \zeta$. « Voici ce que nous avons donné au roi Ramestès, lui qui règne sur toute la terre avec bonheur, qu'Hélios aime, ainsi que le puissant Apollon, ami de la vérité, fils de Hérôn, de naissance divine, créateur de la terre, qu'Hélios a choisi, vaillant fils d'Arès, roi Ramestès, à qui toute la terre est soumise grâce à sa force et à son courage, roi Ramestès fils d'Hélios, qui vit éternellement ${ }^{3}$. » Deuxième colonne. (19)

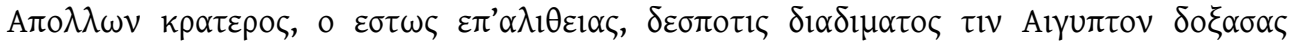

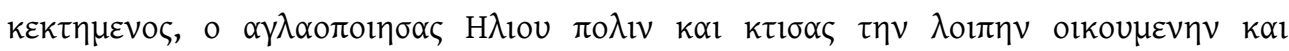




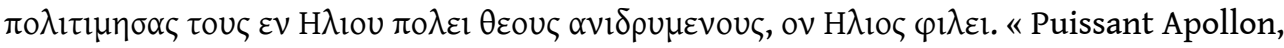
celui qui est établi sur la vérité, maître du diadème, celui qui a glorifié l'Égypte qu'il a acquise, celui qui a illuminé Héliopolis, qui a créé le reste de la terre, qui a comblé d'honneurs les dieux dont les statues se dressent à Héliopolis, qu'Hélios aime ${ }^{4}$.»

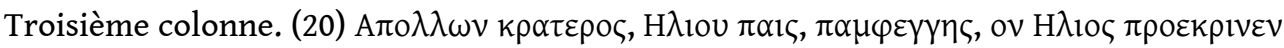

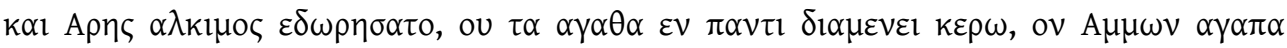

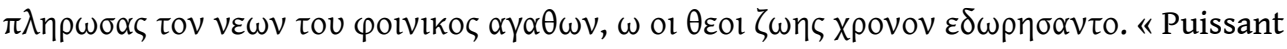
Apollon, fils d'Hélios, tout brillant, qu'Hélios a choisi et que le vaillant Arès a gratifié de présents, dont les bienfaits demeurent en tout temps, que chérit Ammon, celui qui a rempli de bienfaits le temple du Phénix, à qui les dieux ont accordé le temps d'une

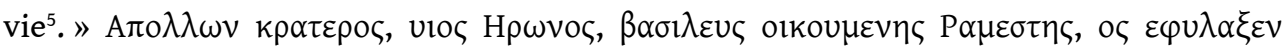

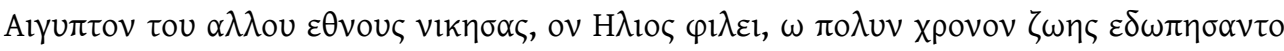
$\theta \varepsilon \circ 1, \delta \varepsilon \sigma \pi о \tau \eta \varsigma ~ o i k o u \mu \varepsilon v \eta \varsigma ~ P \alpha \mu \varepsilon \sigma \tau \eta \varsigma, ~ \alpha l \omega v o \beta$ loc. « Puissant Apollon, fils de Hérôn, le roi de la terre Ramestès, qui a protégé l'Égypte et vaincu les peuples étrangers, qu'Hélios aime, à qui les dieux ont accordé une longue durée de vie, le maitre de la terre

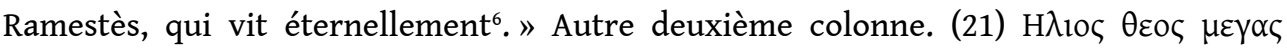

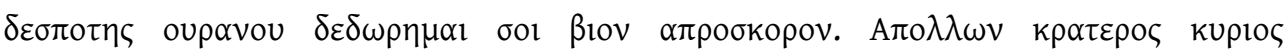

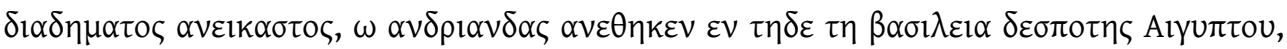

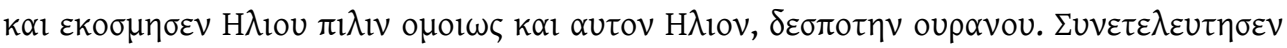

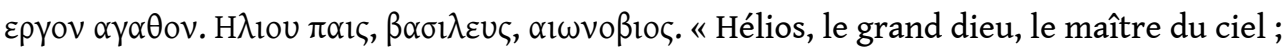
je t'ai donné une vie durable (?) Puissant Apollon, maître du diadème, sans pareil, pour qui le maître de l'Égypte a élevé des statues dans ce royaume, et qui a décoré Héliopolis et pareillement Hélios lui-même, maître du ciel. Il a mené à terme une belle œuvre, le fils d'Hélios, le roi, qui vit éternellement ${ }^{7}$." Troisième colonne. (22) H $\lambda_{1 \circ}$ ऽ $\theta \varepsilon \circ \varsigma$

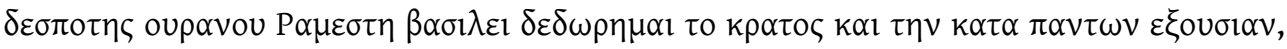

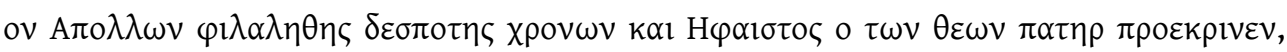

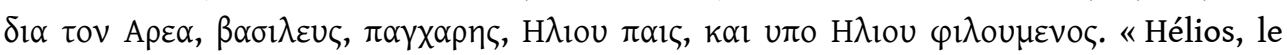
dieu, le maître du ciel, au roi Ramestès : je t'ai donné de la force et du pouvoir sur tout, toi qu'Apollon, ami de la vérité, maître du temps, a choisi, ainsi qu'Héphaïstos, le père des dieux, grâce à Arès, roi plein de joie, fils d'Hélios, et aimé d'Hélios ${ }^{8}$.» Face est,

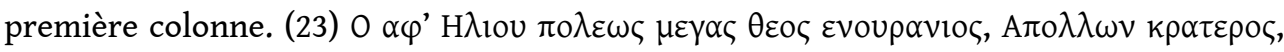

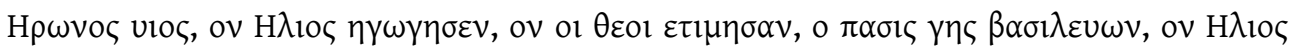

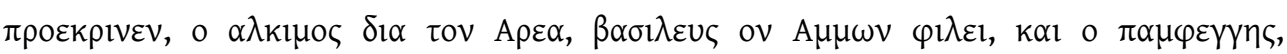

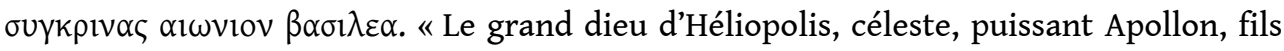
de Hérôn; qu'Hélios a élevé, que les dieux ont honoré, qui règne sur toute la terre, qu'Hélios a choisi, vaillant grâce à Arès, le roi qu'aime Ammon, tout brillant, qui a déterminé le roi éternel ${ }^{9}$. " Jusqu'à la découverte de la pierre de Rosette, ce texte demeurera la seule traduction connue d'une inscription hiéroglyphique. Malheureusement, tant qu'on ne comprend pas les hiéroglyphes, personne ne sait de quel obélisque il s'agit ${ }^{10}$, ce qui interdit de l'utiliser pour obtenir un bilingue grecégyptien qui permettrait un éventuel déchiffrement.

\section{Horapollon}

5 En 1419, Cristoforo Bundelmondi acquiert dans l'île grecque d'Andros, une édition des Hieroglyphika d'Horapollon, un païen du ve siècle originaire de la région d'Akhmim, tardivement converti au christianisme. Sa publication à Venise par Alde Manuce en 
1505 renouvelle l'intérêt pour les hiéroglyphes. Pourtant, son contenu est une catastrophe pour le déchiffrement. En effet, si Horapollon décrit des hiéroglyphes, il n'explique pas véritablement l'écriture hiéroglyphique, car il s'intéresse à la symbolique religieuse des images de son époque, donc de la fin du paganisme, sans dominer toujours pleinement son sujet. L'entrée 26 en fournit un exemple assez simple :

[Comment ils représentent l'(idée d')ouvrir.] Voulant représenter l'(idée d')ouvrir, ils peignent un lièvre, parce que cet animal a toujours les yeux ouverts ${ }^{11}$.

Nous savons aujourd'hui que cette lecture est juste mais que le raisonnement qui l'accompagne est insensé. En effet, le nom « lièvre » wn s'écrit à l'aide de l'idéogramme qui le figure est écrit à l'aide du signe manière d'un rébus. La graphie pleine du verbe composée du bilitère est déterminé par l'idéogramme $\square$ qui représente un battant de porte ou un couvercle. L'entrée 59 est moins évidente à interpréter :

[Comment ils représentent un roi très puissant.] ${ }^{12}$ Quand ils veulent représenter un roi très puissant, ils peignent un serpent disposé comme (pour figurer) l'univers et ils lui mettent la queue dans la bouche. Ils écrivent le nom du roi au milieu de l'enroulement, donnant à entendre par cette graphie que le roi régit l'univers. Le nom que les Égyptiens donnent au serpent est meisi.

Ce qu'Horapollon présente comme un serpent est simplement le cartouche $\square$, une boucle tressée qui entoure les deux derniers noms de la titulature royale, ce que confirme l'entrée 61 :

[Comment ils indiquent le maître du monde. $]^{13}$ Enfin quand ils conçoivent et désignent le roi (en tant que) souverain du monde, ils peignent encore le serpent; mais ils notent à l'intérieur de celui-ci "grande maison" et (ceci) avec raison : car lui <seul> possède un palais dans le monde.

Ce qu'il décrit correspond à la graphie

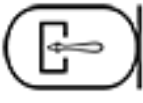
Pr-3 «Pharaon», composée du cartouche $\longrightarrow$ contenant les idéogrammes $\square-$ pour «maison " et " grand ». Elle est fréquente à l'époque romaine pour désigner l'empereur sur les parois des temples égyptiens. De ce fait, il est tentant de rapprocher sa lecture meisi du terme égyptien D'autres entrées sont assez éloignées de l'écriture hiéroglyphique :

[Comment ils représentent un homme pris de fièvre et se guérissant lui-même.] ${ }^{15}$ Voulant représenter un homme pris de fièvre et se guérissant lui-même, ils peignent un lion qui mange un singe : car quand (le lion) a la fièvre, il se guérit en mangeant du singe. 
L'image qu'il décrit évoque le mythe de «La Lointaine». Ce singe est un babouin, une manifestation de Thot $\frac{211}{0411}$

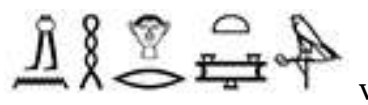
Dhwty qui remplace dans certaines versions Onouris var.

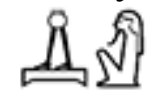

$J n(w)-H r(y) . t$ dont le nom signifie «Celui qui a ramené la Lointaine ». Il raconte des histoires à la déesse la fille de Rê, en lui présentant de la bière colorée avec de l'ocre rouge pour qu'elle croie boire du sang humain, afin de l'enivrer et de la ramener en Égypte. Le thème iconographique existe donc, mais il ne s'agit pas à proprement parler de signes hiéroglyphiques. Quoi qu'il en soit, l'interprétation qu'il livre de cette scène est absolument insensée ${ }^{16}$. Ainsi, pour comprendre Horapollon et en faire bon usage, il faut à la fois savoir lire les hiéroglyphes ptolémaïques, connaître la nature des dieux égyptiens, et leurs mythes à l'époque gréco-romaine. Il va sans dire que les hommes de la Renaissance ne maîtrisent aucune de ces matières. De même, un nombre considérable d'auteurs antiques et byzantins traitent des signes d'écriture de l'Égypte pharaonique. La plupart d'entre eux considère qu'il s'agit de dessins symboliques, énigmatiques, allégoriques, métaphoriques, qui représentent une langue mystérieuse, magique, exprimant des sujets supérieurs qui ne peuvent être dévoilés au commun, certains auteurs précisant même que l'initié peut les lire dans sa propre langue. C'est un piège dans lequel tomberont tous les déchiffreurs. Barthélemy sera le premier à s'en méfier et Champollion sera le seul capable d'en tirer parti. Le terme "hiéroglyphes " n'apparaît qu'à la Renaissance, probablement sous la plume du célèbre Nostradamus

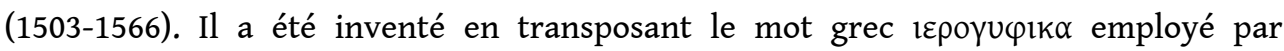
Horapollon $^{17}$. L'ouvrage de ce dernier dissimule un piège supplémentaire. Il était rédigé en copte à l'origine. Son traducteur grec, Philippe, ignorant la culture pharaonique, a ajouté un grand nombre d'entrées, presque toutes ahurissantes, à celles qu'avait rédigées initialement Horapollon. L'entrée 113 en livre un exemple dont les biologistes autant que les égyptologues apprécieront le contenu: "Voulant signifier un homme qui mange sans ménagement les vivres d'autrui et qui consume ensuite les siens propres, ils peignent un poulpe : car celui-ci, quand il est privé de la nourriture qui lui vient d'autrui, mange ses propres tentacules ${ }^{18}$.»

En 1526, Pietro della Valle rapporte d'Orient cinq grammaires et deux lexiques coptearabe. Le copte est la langue parlée par les chrétiens d'Égypte jusqu'au début du $\mathrm{Xv}^{\mathrm{e}}$ siècle. Elle périclite progressivement au bénéfice de l'arabe à partir de l'époque abbasside jusqu'à sa disparition comme langue d'usage à cause de l'épuration ethnique exécutée par les Mamelouks ${ }^{19}$. Cependant, si le copte n'est plus une langue vivante, ce n'est pas non plus une langue morte à proprement parler. En effet, encore aujourd'hui, elle subsiste comme langue d'église, alors que les chrétiens d'Égypte parlent arabe au quotidien. Son usage est comparable à celui du latin en Europe jusqu'au début du $\mathrm{xx}^{\mathrm{e}}$ siècle. L'arrivée de ces ouvrages est un évènement d'une importance capitale pour l'histoire du déchiffrement, car le copte est la langue des Anciens Égyptiens, les hiéroglyphes transcrivant un état antérieur de cette langue. Cependant, en Europe, au $\mathrm{XVI}^{\mathrm{e}}$ siècle, personne ne le soupçonne encore.

Les érudits arabes d'Égypte s'intéressent aussi aux hiéroglyphes ${ }^{20}$ qui couvrent les monuments pharaoniques qu'on pouvait encore voir en grand nombre, suscitant des sentiments divers : haine de la religion païenne, crainte des pouvoirs suscités par ces 
images, envie d'acquérir ces pouvoirs, et surtout de trouver les trésors cachés des pharaons... Un certain nombre de traités en livrent des explications insensées qui reposent sur une pensée ésotérique, cabalistique, analysant les signes, soit comme des mots, soit comme des sons, de façon comparable aux idées exprimées en Europe avant Champollion (fig 2). Ils qualifient les hiéroglyphes de قلام برباوي qalam birbawy « écrit templier pharaonique » à cause de leurs emplacements sur les parois des édifices pharaoniques, dont le nom arabe بربا birbâ est la transcription du copte прпє «le temple" qui dérive de l'égyptien nomment aussi قلام كاهني qalam kahiny «écrit sacerdotal païen», en dérivant le terme قاهن kahin qui s'applique aux prêtres des incroyants. Ils les qualifient aussi de qalam al-tayr « écrit des oiseaux ». Les raisons de cette appellation peuvent être diverses. D'abord, les signes hiéroglyphiques représentant des oiseaux sont nombreux. Ensuite, les Arabes ont peut-être su que les auteurs grecs antiques ${ }^{22}$ racontent que c'est Hermès (c'est-à-dire Thot) qui a inventé l'écriture et qu'en conséquence, l'alphabet des Égyptiens commence avec l'ibis (l'animal du dieu Thot). En effet, les Égyptiens, depuis au moins l'époque perse, classent leurs mots suivant un ordre qu'ils mémorisent grâce à des noms d'oiseaux, à la manière de ce que nous faisons aujourd'hui lorsque nous apprenons l'alphabet (a comme abeille, b comme baleine, etc.). L'ordre suivi par les Anciens Égyptiens n'était pas «alphabétique » (a, b, c, d...), mais «halahamique» $(h, l, h, m . .$.$) . Ainsi, «ibis», 口 11$ égyptien hiéroglyphique, représente le $\mathrm{h}$, première lettre de leur liste ${ }^{23}$. Le plus ancien exemple connu d'ordre halahamique au monde a été découvert en 2015 sur un ostracon de la seconde moitié de la XVIII dynastie, provenant de la tombe thébaine 99, au nom du directeur des choses scellées Sénéfér/Sénéfei ${ }^{24}$. En Égypte, selon la tradition, les Coptes et leur langue sont les descendants des Égyptiens de l'époque pharaonique. Leur

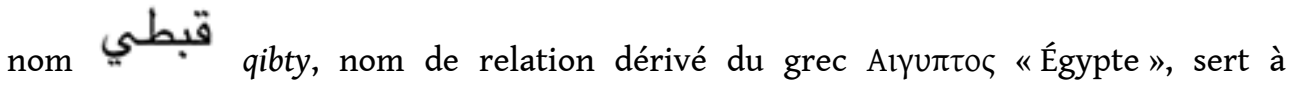
différencier les chrétiens d'Égypte des juifs et des musulmans. C'est pourquoi, les érudits arabes nomment les hiéroglyphes قلام قبطي qalam qibty « écrit copte». Ainsi, en Europe, l'idée de la filiation entre les langues copte et pharaonique trouve probablement sa source dans cette tradition. 


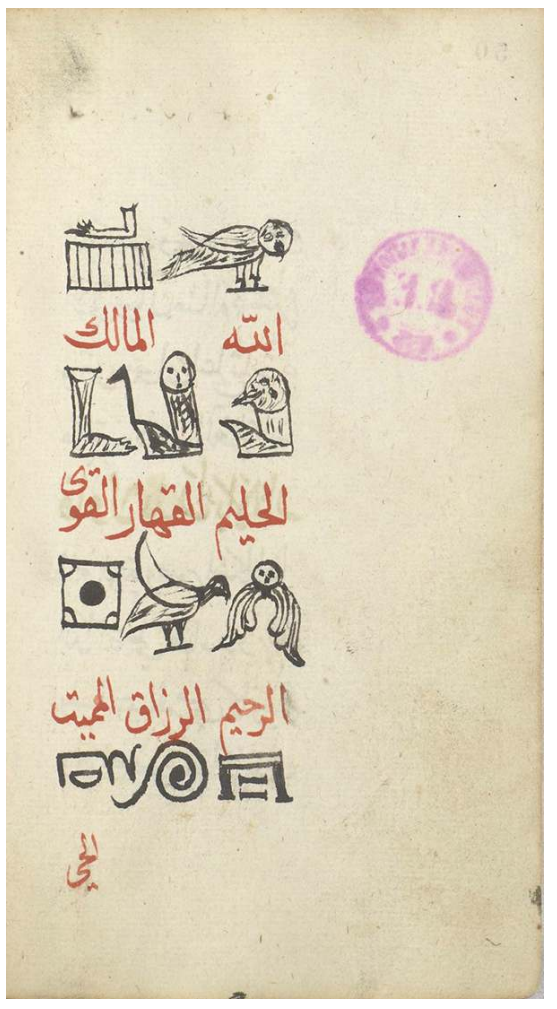

Liste de signes hiéroglyphiques accompagnés d'une valeur idéographique en arabe - D'après Abou Bakr ibn Ahmd Ibn Wahšiyya, Šawq al-mustaham fi ma'rifāt rumuz al-aqlam, 1751, folio 50b - Source gallica.bnf.fr / Bibliothèque nationale de France

7 À la Renaissance, le développement de la diplomatie et de l'espionnage, en Italie d'abord, puis dans toute l'Europe, incite chaque prince à employer un mathématicien qui chiffre son courrier. C'est-à-dire qu'à l'aide d'un code, il le rend incompréhensible à ceux à qui ce courrier n'est pas destiné. À l'inverse, ce mathématicien décrypte le code $\mathrm{du}$ courrier des autres princes. Ce sont ces mathématiciens qui analysent les hiéroglyphes en suivant les méthodes utilisées pour déchiffrer les courriers secrets, d'où l'origine de l'expression « déchiffrer » une écriture antique.

\section{Blaise de Vigenère}

La pensée du diplomate Blaise de Vigenère (1523-1596) reflète parfaitement les idées de cette époque, mêlant rationalité, inspiration des philosophes grecs - en particulier le Cratyle de Platon - et pensée cabalistique, alchimique et ésotérique. Dans son Traicté des chiffres, ov secretes manieres d'escrire ${ }^{25}$, il s'intéresse principalement à la cryptographie, expliquant une grande variété de méthodes de chiffrement, mais il élargit sa réflexion à des questions plus générales concernant langage et écriture. Ainsi, il traite des alphabets de différents peuples, dont en quelques phrases il présente la position géographique, la religion et quelques coutumes. Il montre la différence entre écriture ordinaire et écriture secrète, déguisée, considérant cependant qu'il s'agit toujours d'un vrai chiffre, de même que les actions des hommes; et qu'en fait

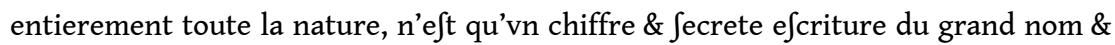

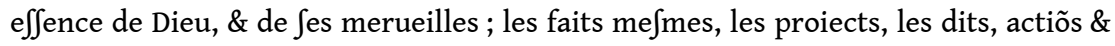
comportemens des humains, que Jont ce pour la plus grand-part finon chiffre ${ }^{26}$ ? 
Vigenère s'interroge sur la relation entre le langage pensé, parlé et écrit, et de là, sur la question des liens entre langue et écriture ainsi que sur leur évolution. Il argumente en faveur de la supériorité du langage écrit, pérenne, sur le langage parlé, éphémère, estimant :

que l'efcriture eft plus Spirituelle que la parolle, qui tient plus du corps, car elle tombe fouz le Jentiment de l'oye, plus groffier \& materiel que celuy de la veuë, ouquel confifte l'efcriture ${ }^{27}$.

10 Il considère que la langue originelle est l'hébreu, ainsi que son écriture, inventée avant même la création par Dieu qui l'a enseignée à Adam dès le Paradis, et que toutes les langues et écritures en dérivent ${ }^{28}$.

S'il y a quelque langage perceptible que parlent les Anges, ce deuroit eftre l'Hebraïque, attendu que ç'a efté le premier de tous, \& qui emana de la propre bouche de Dieu au premier homme (...) les cabaliftes tiennent que les lettres Hebraiques doiuent eftre auffi les premières; \& qu'elles furent forgees au ciel quant \& le parler que Dieu enfeigna à Adam, aiant efté creé de luy en aage parfait, apte \& capable de parler tel langage qu'on luy apprendroit ${ }^{29}$.

Pour commancer donques aux caracteres hebraïques, qui Jont les plus anciens de tous, voire formez du propre doigt du Jouuerain Dieu, auant la creation du fiecle, $\mathrm{fi}$ nou-nous en voulons raporter à ceux que nous auons là de $\iint u s$ alleguez cy deuant $(. . .)^{30}$.

11 Selon lui, les lettres ont changé de forme en fonction des aléas de l'histoire, de la nature des utilisateurs de l'écriture et de sa finalité. Dans ce contexte, les variations des signes hébraïques sont bien entendu tributaires des relations entre les juifs et Dieu.

Il y a un autre Rabi qui afferme, que dés le commancement la loy fut donnee et efcrite és mefmes langue et caracteres qu'on voit encore pour le prefent, mais que pour la preuarication des Ifraëlites, lors qu'ils vindrēt à Se Jeparer de Iudah, cefte efcritue fut changee en vne autre, et puifapres eftans venus à Je reconoiftre, et faire penitence de leur mefait, l'ecriture premiere leur fut reftablie: Toutesfois Rabbi Simon fils d'Eleazar maintient, que le langage ne l'efcriture ne furent onques changez, ny autres que ceux qu'on pratique pour le jourd'huy ${ }^{31}$.

Concernant l'usage de l'hébreu et les modalités de l'évolution d'une écriture à l'autre, au milieu d'explications erronées se trouvent quelques remarques d'une perspicacité étonnante de la part d'un auteur aussi ancien, qui nous autorisent à considérer Vigenère comme un précurseur des savants du siècle des Lumières.

Dauantage les Hebrieux n'aians point proprement de voyelles, font Jeruir ordinairement ain pour o, non Jans myftere: car comme ain Jignifie non, ou rien,

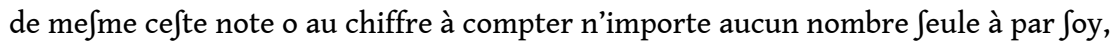
fi elle n'eft accompagnee de quelqu'vne des neuf. Au Jurplus ce caractere denote l'œil, que le Roy doit auoir à tout ; \& par tout : \& Juiuant cela les Egyptiens en leurs

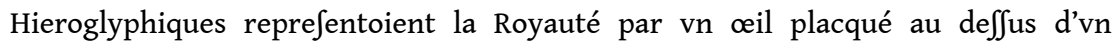
Sceptre, ainfi que le Specifiēt Orus \& Plutarque au traité d'ofiris. Le Sceptre Jignifiant la force, authorité \& pouuoir, \& l'œil la preuoiance ${ }^{32}$.

13 Aucune entrée d'Horapollon ne correspond à une représentation de la royauté par un œil placé au-dessus d'un sceptre, en revanche cela correspond effectivement à la description du nom d'Osiris par Plutarque qui confond sceptre et trône :

(...) les Égyptiens représentent Osiris, leur seigneur et roi, par un œil et un sceptre. Quelques-uns prétendent aussi que ce nom signifie : "qui a beaucoup d'yeux", attendu que os en langue égyptienne veut dire beaucoup, et iri, œil ${ }^{33}$. 
Les explications de Plutarque sont fausses, mais sa description renvoie à la graphie

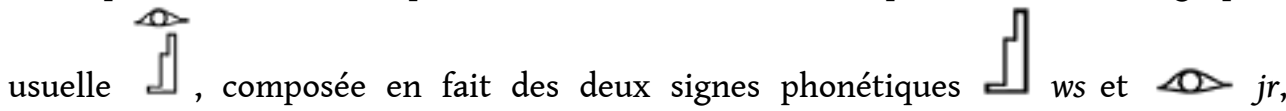

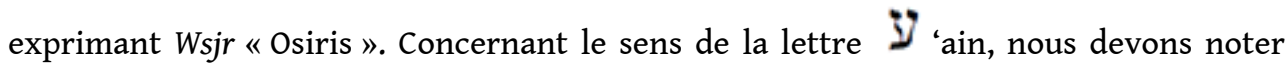
que 'ain « rien » s'écrit de celle de la lettre gutturale $\grave{Z}$ 'ain. En conséquence, même si elle est très astucieuse, l'explication de Blaise de Vigenère ne tient pas. Cependant, il exprime ici deux remarques considérables : d'une part, le fait que le nom de la lettre $\mathbf{Z}$ 'ain «dénote l'œil »; d'autre part, le fait que puisque l'hébreu n'a « point proprement de voyelles », la lettre 'ain sert pour «o 0 . Pour que ces deux réflexions prennent tout leur sens, il faudra attendre le déchiffrement du phénicien par Barthélemy ${ }^{34}$ en 1758, puis en 1916, celui de l'écriture protosinaïtique par Gardiner ${ }^{35}$ et sa découverte de la genèse et de la diffusion de l'alphabet. Ainsi, nous savons aujourd'hui que Blaise de Vigenère - en suivant les auteurs antiques Pline et Eusèbe - avait déjà pressenti en partie la nature des relations des alphabets entre eux :

Ainsi Moyfe eut deux fortes de caracteres, l'vne pour les chofes facrees, affauoir l'Hebraïque, qui eft celle que nous auons; \& l'autre pour les prophanes, cõme la Iuftice, police, cõmerces, \& Semblables affaires du monde ; \& pourtant vulgaire, \& vfitee de tout le peuple Iudaïque ; qu'on tient eftre la Samaritaine, celle dont vfoient les anciens Chaldees, \& qui $\int e$ communica depuis aux Pheniciens: Dont tout ainfi que de l'Hebraïque Sont prouenues la Syriaque \& l'Arabe fque, fut enfantee la Grecque ; \& cõfequemment la Latine, qui confifte toute ou peu s'en fault des capitales Grecques, comme on peult voir $(. . .)^{36}$.

Ainsi donques que la langue Chaldee \& la Syriaque font venues de l'Hebraïque, en Semblable de l'efcriture Samaritaine Sont procedez les caracteres Pheniciens, dont les Grecs ont pris la plus-part des leurs : Et par confequant les Latin emprunté ceux des Grecs, comme il a efté dit cy deffus ${ }^{37}$.

Comme argument, il constate avec raison la similitude des noms qui désignent les lettres des différents alphabets :

Ce que deffus confirme encore Eufebe par la propre denomination des Grecques; où à l'imitation du Chaldaîfme a efté adioufté à la plufpart vn a pour leur definence, auec quelques tranfpofitions en d'aucunes, comme Alpha au lieu d'Aleph; Betha pour Beth ; Gamma pour Gamel, ou gimel ; Delta pour Daleth, etc. ${ }^{38}$

En s'appuyant sur les écrits de saint Jérôme, il utilise déjà les considérations qui permettront deux siècles plus tard ses déchiffrements à Barthélemy :

que les caracteres Samaritains furent les premiers qu'eut le peuple Hebrieu (...) Que les Hebrieux euffent vingt-deux lettres en leur alphabet, la langue mefme Syriaque le temoigne asfez, qui pour la plus part est fort aprochante de l'Hebraïque ; ayant auffi vingt-deux lettres, et d'un mefme nom, mais de differends caracteres $(. . .)^{39}$.

Enfin, il exprime, bien avant Athanasius Kircher, l'idée selon laquelle le nom des Coptes vient de la ville de Koptos :

«Les Cophtites ont l'appellation de leurs lettres tout de me $m e$ que les Iacobites, mais les caracteres Jont aucunement differends, en nombre aufji de trente-deux. Poftelle en fon traicté des lettres pheniciennes les confond auec les Iacobites, combien qu'ils soient plus particulierement Égyptiens; \& Chreftiens de plus longuemain : dits ainfi au refte de la ville de Coptus, dont parle Plutarque au traicté d'Ofyris ; \& Pline liu.5.chap.9. qui luy attribue l'vn des Sieges \& gouuernemens de l'Egypte $^{40}$. 
En fait, son explication est erronée car le nom des Coptes est une déformation du grec

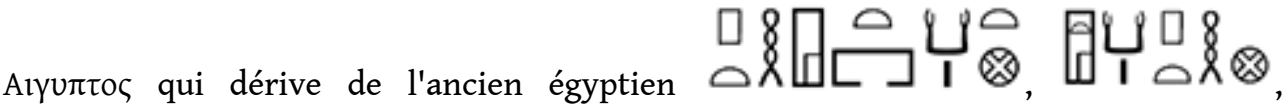
0810
$0 \times 1$

Hw.t-k3-Ptḥ (Houtkaptah), «le Château du ka de Ptah », désignation du temple de Ptah à Memphis et partant, de la ville elle-même ${ }^{41}$.

Quoi qu'il en soit, au XVI ${ }^{\mathrm{e}}$ siècle, à cause de l'influence pernicieuse des auteurs antiques, personne ne peut imaginer que les hiéroglyphes transposent un idiome avec une grammaire spécifique qu'il faut cerner avant de chercher la valeur de ces signes, que l'on croit alors purement symboliques. On considère les hiéroglyphes comme les images héraldiques qui constituent les devises. Blaise de Vigenère ne fait pas exception :

Car les Hieroglyphes des Égyptiens (...) ne Jont à proprement parler, qu'vne maniere de chiffres ; Bien eft vray que nompas diftincts en lettres, Jyllabes, \& dictions, pour en teiftre particulierement vne claufe ; ains certaines marques \& notes comprenans chacune endroit foy quelque Jens entier; ainfi que font à peu-pres nos deuifes, dont elles Jont fort approchantes; pour reprefenter quelque myftere de la diuinité, ou Secret de nature ; ainfi qu'on peult veoir en Orus Apollo, Chæremon, \& autres ${ }^{42}$.

Vigenère est persuadé qu'à côté de ces hiéroglyphes devait exister une écriture profane :

QVANT aux Égyptiens peuple $\int \mathrm{i}$ ancien \& $\int \mathrm{i}$ renommé en toutes fortes d'arts \&

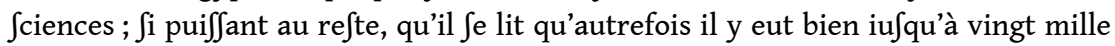
villes habitees, là où pour le iourd'huy à peine $\int \mathrm{y}$ en trouue il cinq ou $\int \mathrm{ix}$, avec dix ou douze bourgades, \& en outre $\int \mathrm{i}$ belliqueux, qu'ils ont eftendu leurs conqueftes, \& donné la loy à la plus grand' part de la terre, il ne faut point faire de doute qu'ils n'aient eu vn langage à part, \& vne efcriture, non tant feulement des notes \& marques qu'on appelle les Hieroglyphiques, leur Seruans de chiffre Jecret refervé aux Rois \& aux Preftres, mais de lettres communes au $\int \mathrm{i}$, dont $\int \mathrm{e}$ peut former vn contexte exprimant par le menu lettre à lettre toutes fortes de conceptions ${ }^{43}$.

Aujourd'hui, il va sans dire que l'écriture hiéroglyphique n'a rien à voir avec un message chiffré puisqu'il s'agit d'une écriture courante, mais juger les Anciens à l'aune des connaissances dont nous disposons serait une erreur épistémologique.

Cependant, d'autres évènements de la Renaissance délivrent des informations qui obligeront les penseurs de cette époque à remettre en cause une partie de leurs convictions. On découvre les «Indes occidentales ", dont les habitants utilisent des écritures non alphabétiques et figuratives, ce qu'on appelle alors "peintures mexicaines ». Des informations les concernant, souvent imparfaites, parfois erronées, arrivent en Europe. Ainsi, par exemple, en 1566, lors de son séjour en Espagne afin d'être jugé pour son application particulièrement cruelle de l'Inquisition au Yucatan, le missionnaire Diego de Landa (1524-1579) rédige une Relación de las cosas de Yucatán qui livre aux $\S 34,40$ et 41 des informations cruciales sur les glyphes mayas, mais qui ne sera éditée qu'en 1864 à Paris. La publication en 1590 de la Historia natural y moral de las Indias, du père jésuite José de Acosta (1539-1600) est traduite en français dès 1598, en anglais en 1604, puis en flamand et en allemand. La rapidité de la traduction de ce livre et de sa divulgation dans toute l'Europe est considérable. L'expansion de l'imprimerie, plus précisément de la typographie, inventée vers 1440 par Gutenberg, joue un rôle d'une importance capitale dans la communication de ces découvertes et des idées qui en découlent. Les relations commerciales et politiques avec l'Extrême-Orient se 
développent, on étudie les langues locales ainsi que les signes qui les transcrivent, totalement étrangers aux systèmes alphabétiques.

21 L'idée que l'on se fait de ces écritures lointaines alimente la réflexion concernant la notion d'écriture. Les signes chinois, alors considérés comme non figuratifs, concis et précis, révélateurs d'un degré élevé d'évolution, sont opposés aux peintures mexicaines figuratives, correspondant à un degré primitif de l'écriture, quand elles ne sont pas envisagées comme de simples signes qui ne soient pas réellement scripturaux. À ce propos, Blaise de Vigenère qualifie les signes chinois et japonais de hiéroglyphiques :

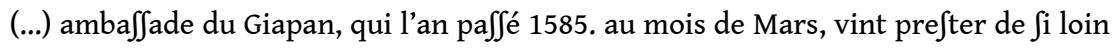

(...) la Chine, \& au Cathaj, dont l'art de l'Imprimerie eft venue à nous ; pour le moins elle leur a efté long temps auparauant cogneuë, il y a deux efpeces d'efcriture, de mefme qu'Apulee racompte d'Egypte ; l'vne de lettres hieroglyphiques ; \& l'autre de lettres communes, qui par leurs afsemblemens peuuent former tous les mots qu'on veult exprimer: (...) La premiere, qu'ils appellent l'efcriture de la Chine, eft commune generalement à toutes les regions des Indes (...) mais employee feulement

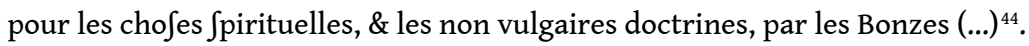

Ces lettres donques hieroglyphiques de la Chine, \& de Chataj, Jont tres-difficiles d'apprendre à lire, \& à former; parce qu'elles confiftent de diuerfes figures de beftes, oifeaux, arbres, herbes ; \& en fomme de tout ce que la nature produift, qui leur sert de notes ${ }^{45}$.

(...) Ce qui nous monftre que fur les notes generales de cefte hieroglyphique efcriture, en les deguifant \& accouplant de diuerfes manieres, fe peuuent former des caracteres composez tous nouueaux $(. .)^{46}$.

Ces remarques sont à l'origine d'idées concernant des relations entre l'Égypte et la Chine qui se développeront jusqu'à ce que Champollion déchiffre les hiéroglyphes; idées qui auront un rôle à jouer, étonnant, dans son entreprise de déchiffrement. Les observations de Vigenère sont aussi les prémices de théories concernant l'évolution des écritures depuis le figuratif jusqu'à l'abstrait, qui influencent encore la pensée actuelle. Cependant, aux XVI et $\mathrm{XVII}^{\mathrm{e}}$ siècles, tous les lettrés sont persuadés que les hiéroglyphes constituent une écriture "philosophale» qui parle au cœur du philosophe ou de l'alchimiste sans avoir besoin d'être prononcée. Le mot alchimie vient de l'arabe الخمية

الكمية / al-khimiya'

al-kimiya', lui-même dérivé du nom de relation égyptien kmy formé sur la même racine que «Égypte»; en copte XHMI, KHM€ $_{47}$.

\section{Athanasius Kircher}

Le jésuite allemand Athanasius $\operatorname{Kircher}^{48}(1602-1680)$ est un savant qui s'intéresse aux domaines les plus variés : mathématiques, physique, optique, astronomie, géographie, histoire, sciences naturelles, théologie... Kircher connaît un nombre inégalé de langues et d'écritures. Il est impensable pour un érudit de cette valeur de délaisser la question des hiéroglyphes. Kircher croit qu'il s'agit d'une écriture secrète réservée aux philosophes, constituée de symboles seulement compréhensibles lorsqu'on a le cœur pur. Bien entendu, c'est insensé et ses résultats font sourire aujourd'hui. Ainsi, il analyse la figure $3^{49}$ en décrivant les signes un par un : « circulus, brachium, ara, catena, brachium, vas niloticum, quid in tabula sacra signent " «cercle, bras, autel, chaîne, bras, vase nilotique, qui se trouvent dans la table sacrée", puis en proposant une interprétation ahurissante: "Diuini beneficia Osiridis, mediantibus sacris, \& catenà 
Genorium procuranda sunt, et Nili beneficia obtineantur ", c'est-à-dire "Les bienfaits du divin Osiris doivent être procurés par le moyen des cérémonies sacrées et de la chaîne des génies afin que les bienfaits du Nil soient obtenus ", alors qu'en fait, il s'agit

$\mathrm{du}$ quatrième nom de la titulature du roi Apriès

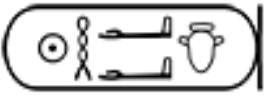
homme de son époque, comme le reflète l'article du Dictionnaire de l'Académie française, publié en 1694 :

HIÉROGLYPHE. s.m. Symbole, caractère, figure qui contient quelque sens mystérieux \& dont les anciens Égyptiens se servoient dans leur Religion \& dans leur Morale. Hiéroglyphes ingénieux. Toute la Théologie des Égyptiens estoit exprimée par des hiéroglyphes, enveloppée sous des hiéroglyphes. HIÉROGLYPHIQUE. adj. de tout genre. Qui appartient à l'hiéroglyphe. Caractère hiéroglyphique, figure hiéroglyphique, symbole hiéroglyphique. On s'en sert aussi très-souvent dans le substantif, \& alors il signifie la mesme chose qu'hiéroglyphe.

Fig. 3

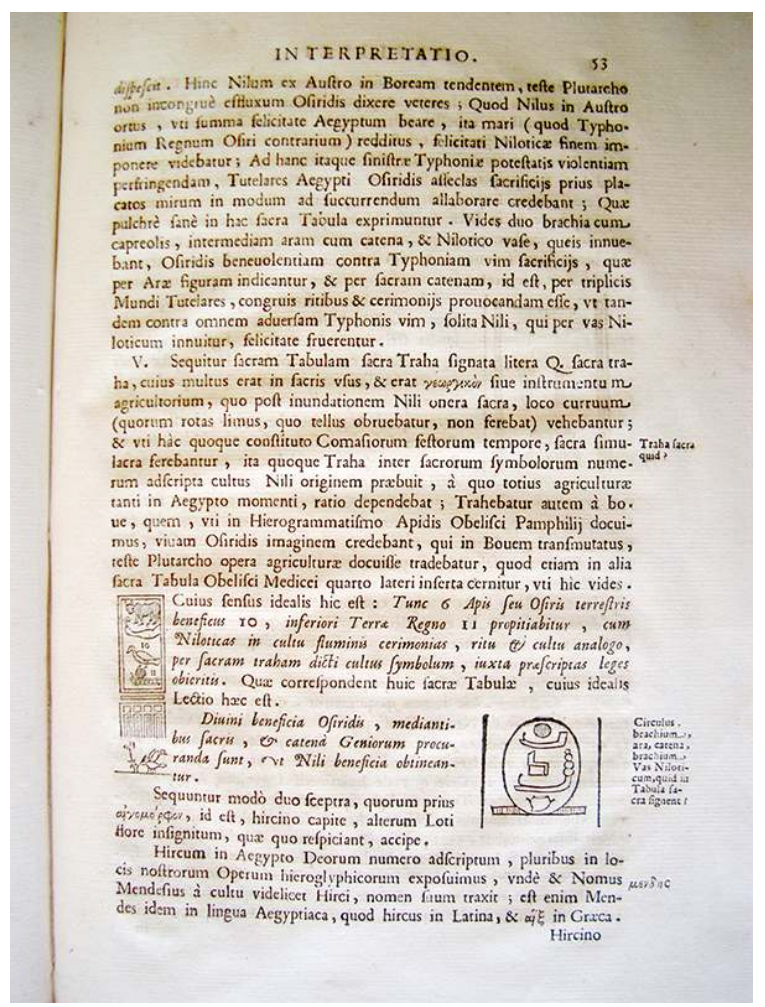

Cartouche d'Apriès - D'après Athanasius Kircher, Obelisci Aegyptiaci nuper inter isaei romani rudera effossi interpretatio hieroglyphica, Rome, Varesi, 1666, p. 53 - Bibliothèque universitaire d'Heidelberg C $2901 \mathrm{FOL}$

À la fin de sa vie, Kircher a édité neuf ouvrages concernant l'Égypte ancienne, sans avoir rien percé du secret des hiéroglyphes. Cependant, il a posé un premier jalon pour la science égyptologique à venir grâce à ses travaux sur la langue copte. En 1636, il publie le Prodromus coptus sive cegyptiacus, qui est le premier ouvrage scientifique traitant de la langue copte et, en 1644, le lexique de copte Lingua cegyptiaca restituta (Fig. 4) résultant de ses travaux sur les écrits rapportés par Pietro della Valle. Il suppose, suivant sur ce point Nicolas Fabri de Peiresc (1580-1637), qu'il a rencontré en 1633, que les Anciens Égyptiens parlaient le copte, ayant constaté qu'il peut expliquer 
les noms transmis par les auteurs classiques à l'aide de cette langue. Il est probable que cette idée soit parvenue en Europe par le truchement des érudits arabes, peut-être grâce aux informateurs orientaux de Peiresc. Cependant, Kircher conserve la croyance de la Renaissance, selon laquelle les Coptes tirent leur nom de la ville de Koptos...

Fig. 4

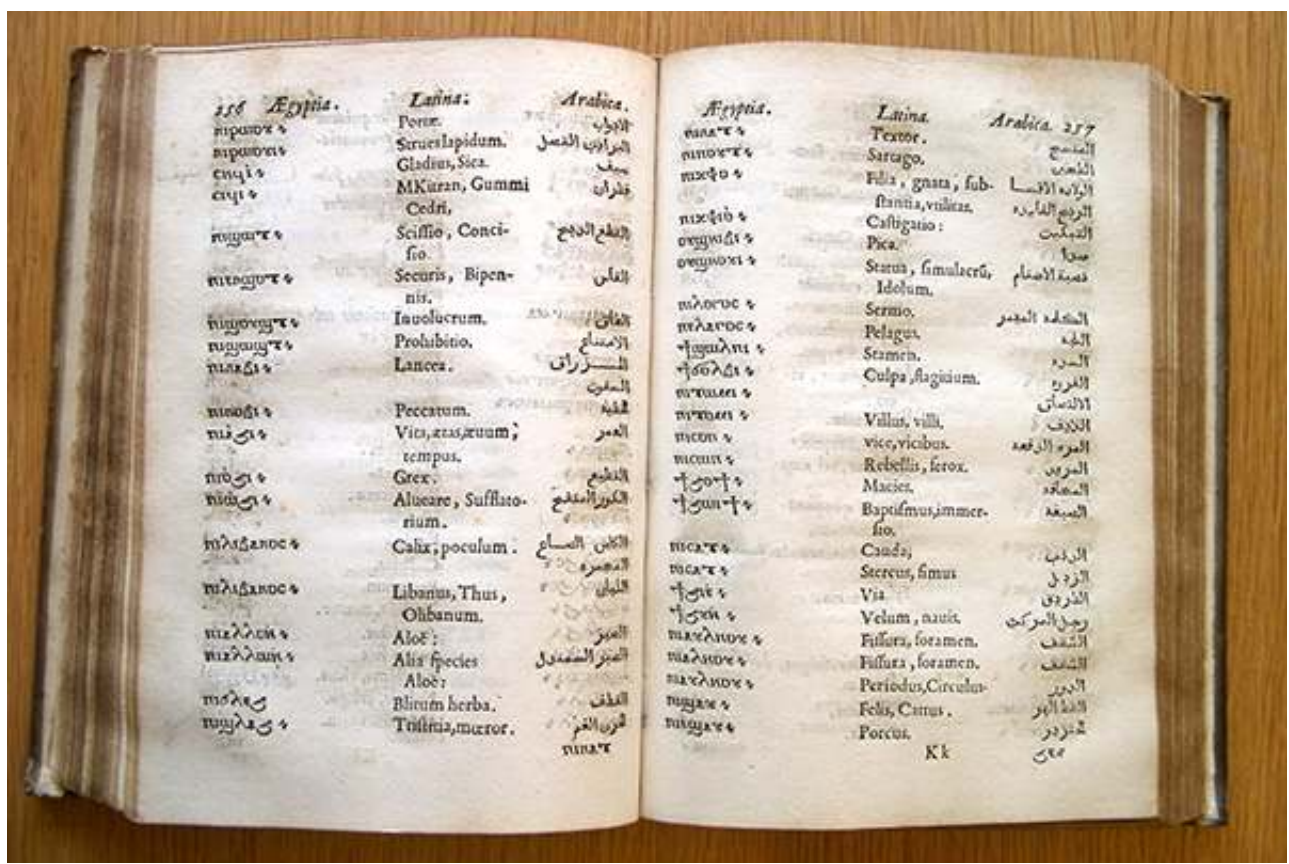

Dictionnaire arabo-copto-latin - D'après Athanasius Kircher, Lingua Aegyptiaca restituta. Opus Tripartum, Una cum Supplemento a D Ferdinandum III. Rom. Imp., Rome, 1644, p. 256-257 - Bibliothèque universitaire d'Heidelberg ML 84 A 7600

Le fait qu'il travaille à partir de copies de vrais monuments et non d'inventions fantaisistes constitue la différence fondamentale entre Kircher et les déchiffreurs de la Renaissance. En témoigne la plus ancienne image connue du groupe statuaire Louvre $\mathrm{A} 47^{50}$ représentant trois grands-prêtres de Ptah de la XII ${ }^{e}$ dynastie, exposé dans la galerie d'étude du Moyen Empire (fig. 5). Ce groupe présente toutes les caractéristiques (formules spécifiques et massivité) des monuments de particuliers placés dans l'aire des temples divins, habituels au Moyen Empire (fig. 6). Le bloc est brisé et le personnage de droite a disparu. La gravure de Kircher prouve que c'était déjà le cas en 1681. Cependant, quelques éléments brouillent les pistes pour la reconnaissance de l'œuvre. D'abord, le dessinateur de Kircher n'a reproduit qu'un des deux personnages subsistant puisqu'ils sont similaires. Ensuite, il a copié le texte en l'inversant, c'est-à-dire signes tournés vers la gauche, alors qu'ils sont orientés vers la droite sur le groupe du Louvre. Enfin, les inscriptions ne sont pas exactement celles de la statue du Louvre. En fait, le dessinateur a souvent interprété les signes: par exemple, d'une bouche ou d'une corbeille il a fait un poisson, il a remplacé l'abeille par un rapace. De plus, n'ayant pu insérer tous les signes dans la place impartie, il en a déplacé quelques-uns qui se trouvent désordonnés de fait. Ainsi, cette copie est assez approximative et même fantaisiste, rendant ce texte inutilisable pour les déchiffreurs. Pourtant une étape est franchie, car aujourd'hui n'importe quel égyptologue peut la lire, en fournissant un petit effort, mais sans grande difficulté (fig. 7). 
Fig. 5

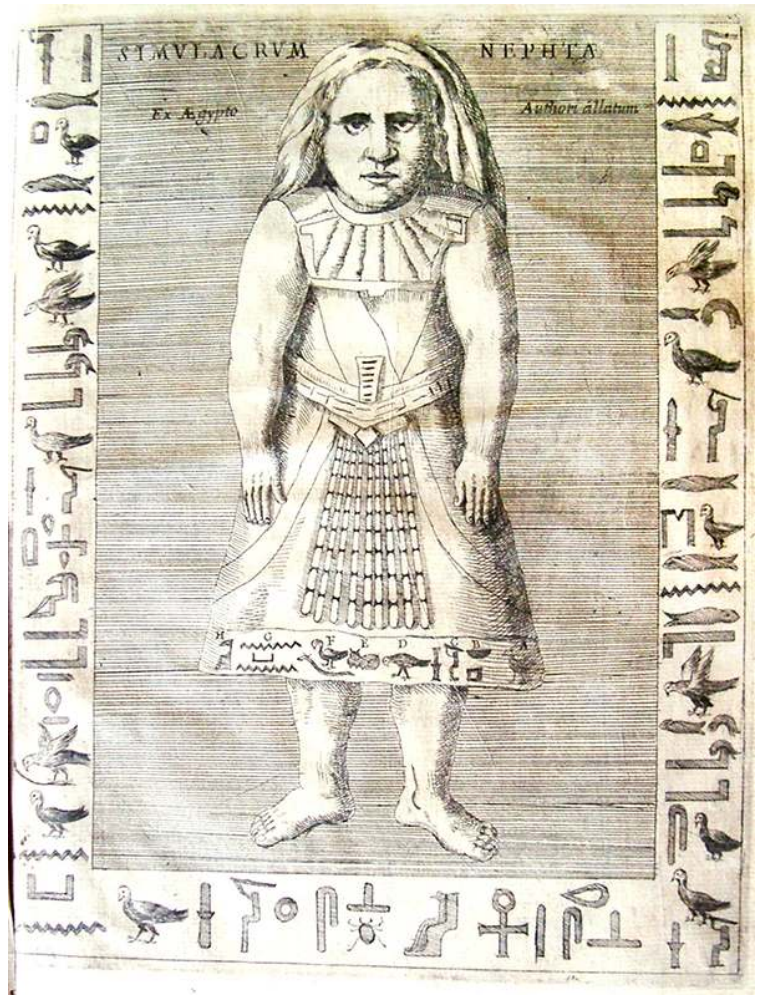

Grand-prêtre de Ptah - D'après Athanasius Kircher, Obelisci Aegyptiaci nuper inter isaei romani rudera effossi interpretatio hieroglyphica, Roma, 1666, face à la p. 136 - Bibliothèque universitaire d'Heidelberg C 2901 FOL 
Fig. 6

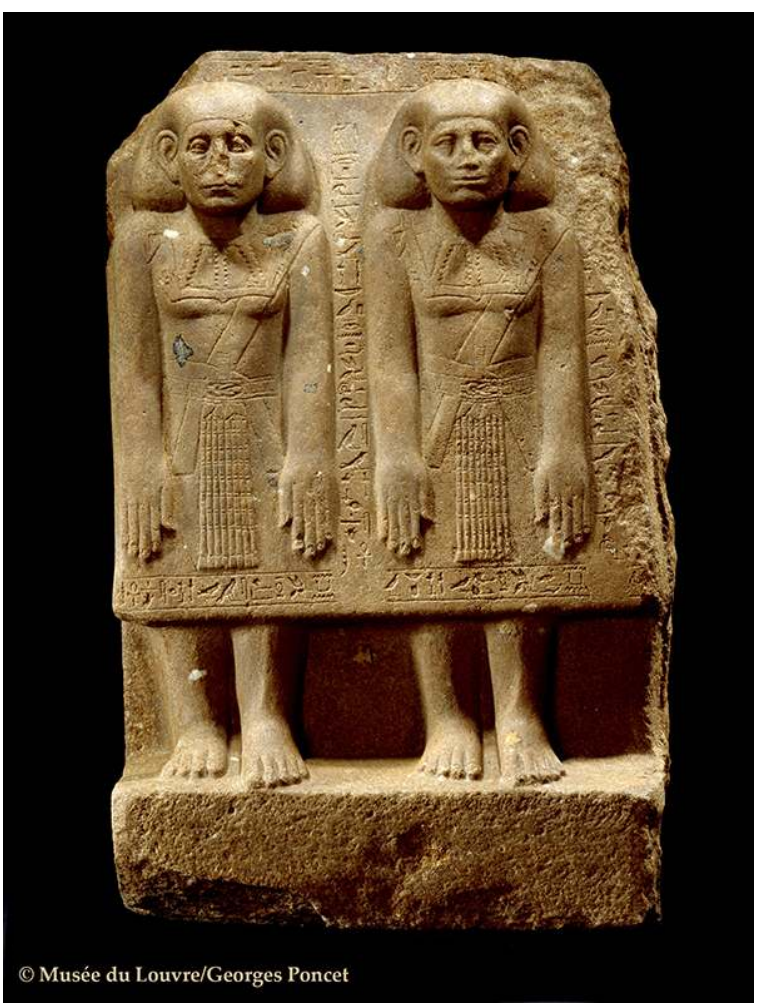

Deux grands-prêtres de Ptah : Sehetepibrê-ankh-nedjem et son fils Nebpou

Règne de Sésostris III (1862 - 1843 avant J.-C. - Musée du Louvre, département des Antiquités égyptiennes, Inv. A47

(c) Musée du Louvre/Georges Poncet 
Fig. 7

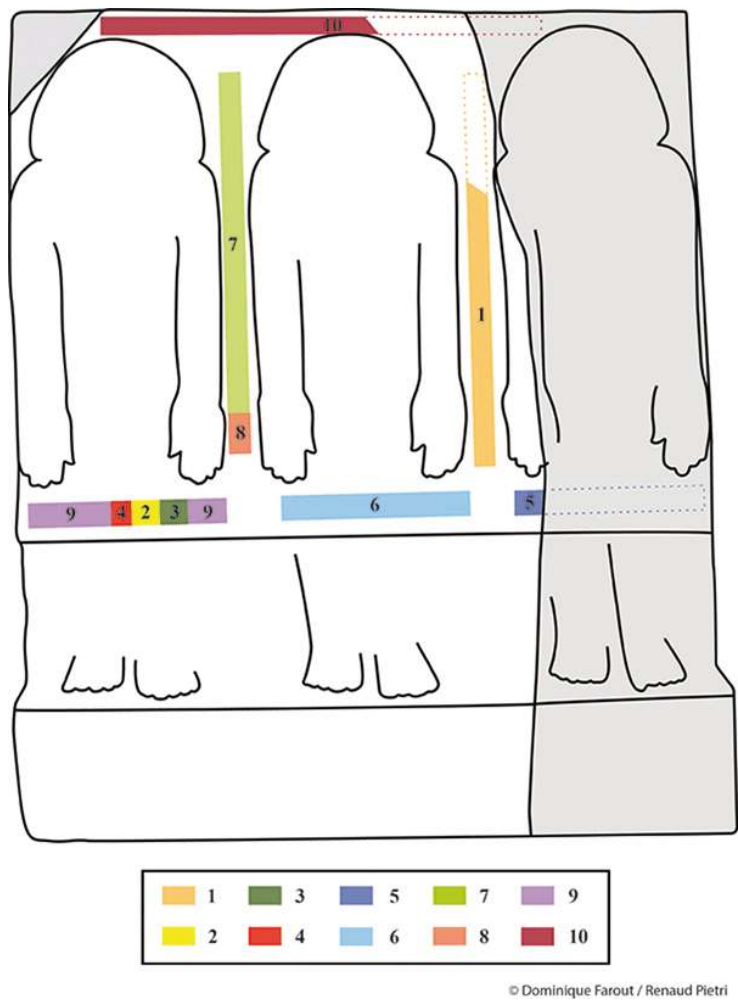

Le groupe A47 reconstitué. Les numéros renvoient à l'étude de la copie du livre de Kircher.

(c) Dominique Farout / Renaud Pietri

La colonne de gauche est en fait à droite sur l'original et le tiers supérieur manque :

(1)
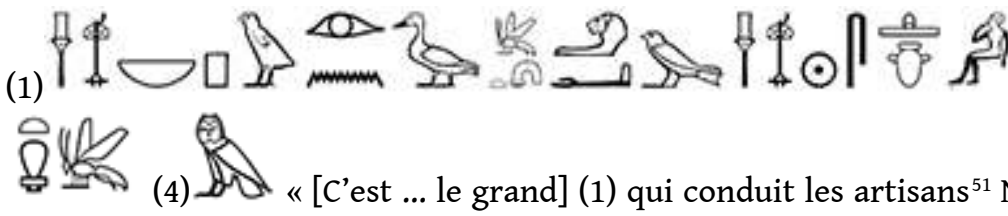

(4) 1 " [C'est ... le grand] (1) qui conduit les artisans ${ }^{51}$ Nebipou $^{52}$ qui a fait (ceci) pour (son) fils, le chancelier du roi de Basse Égypte, gouverneur, grand qui conduit les artisans, Séhétepibrê le jeune. » La fin de la colonne se trouve en réalité sur le pagne du personnage de gauche: «(2) gouverneur, (3) chancelier du roi de Basse Égypte, (4) prêtre-[se]m ».

L'inscription horizontale sur la partie inférieure du pagne de la gravure correspond à la partie subsistante de celle du pagne du personnage disparu à droite : (5) «... Séhétepibrê] le jeune ", suivie de celle du personnage central: (6)

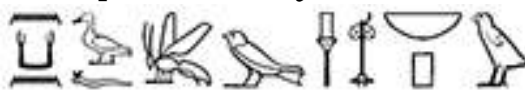

"pour le ka de son fils, le (chancelier du) roi de Basse Égypte, (le gouverneur), le grand qui conduit les artisans, Nebipou ». On notera que le copiste a oublié les signes of et «gouverneur».

L'inscription verticale de droite est à gauche sur l'original, c'est-à-dire entre les deux personnages subsistants : 
(7)

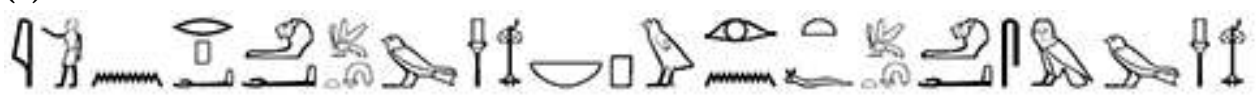

« (7) C'est le prince gouverneur, chancelier du roi de Basse Égypte, Nebipou qui a fait (ceci) pour son père le chancelier du roi de Basse Égypte, gouverneur, prêtre-sem, le grand qui conduit les artisans. » La fin de la colonne $\odot$ manque. Elle est en partie reproduite à la fin de la ligne horizontale inférieure : (8) $\odot$ «(2) Séhétep(ib)Rê-(ânkh-nédjem) ».

Le reste de la ligne horizontale inférieure correspond à l'inscription du pagne du personnage de gauche du groupe original : (9)

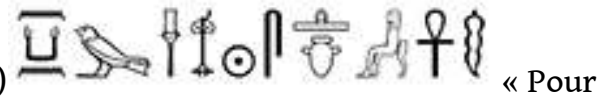

le ka du » (le graveur a déplacé : (3)

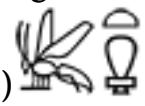

(2)

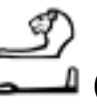

(4) « chancelier du roi de Basse Égypte, gouverneur, prêtre-[se]m » à la fin de la colonne de gauche) « grand qui conduit les artisans Séhétépibrê-ânkh-nédjem ». Le signe ne se trouve pas sur l'original. Le dessinateur a ignoré la ligne fragmentaire d'inscription (10) qui subsiste sur la plaque dorsale au-dessus de la tête des personnages.

Enfin, Kircher est le premier à proposer que l'écriture hiératique soit une cursive des hiéroglyphes, remarque fondamentale. Ainsi, héritier des idées de la Renaissance, Kircher est à la fois un savant à la grande rigueur scientifique et un rêveur aveuglé par les idées symbolistes qu'il faut replacer dans son époque pour apprécier la place de précurseur qu'on ne peut lui dénier.

\section{Le siècle des Lumières : Warburton, Barthélemy, la pierre de Rosette}

Au cours des $\mathrm{XVII}^{\mathrm{e}}$ et $\mathrm{XVIII}{ }^{\mathrm{e}}$ siècles, l'appréhension des hiéroglyphes égyptiens s'éloigne de celle de la devise. On commence à les considérer comme une écriture, et on les rapproche tantôt des peintures mexicaines, tantôt des signes chinois. Il en résulte un intérêt concernant l'invention, l'évolution de l'écriture et la comparaison des systèmes entre eux. La notion d'évolution des systèmes d'écritures, et non pas seulement de signes alphabétiques, est un pas décisif concernant la compréhension du sujet. Cependant, elle est associée à l'idée de valeur et de progrès - avec le sens d'amélioration - de ces écritures et des sociétés qui les génèrent, dont l'influence s'exerce bien au-delà du déchiffrement des langues anciennes. Cette pensée, caractéristique du siècle des Lumières, aura des conséquences malheureuses jusqu'à notre époque.

Des acteurs de cette quête à l'origine de l'orientalisme ${ }^{53}$, nous ne citons que les plus importants. Nicolas Trigault (1577-1628) publie en 1615 De christiana expeditione apud Sinas suscepta ab Societate Jesu, traduit en français en 1616. Il y expose que la façon d'écrire et de composer des Chinois « est semblable à ces figures hiéroglyphiques des Égyptiens». Francis Bacon (1561-1626) s'intéresse en particulier aux moyens de communication extérieurs à l'oralité, comme le langage des sourds-muets, mais aussi 
aux signes chinois qui expriment une relation directe et exacte entre le signe et l'idée. Ainsi, il conclut que le son exprimé par la parole n'est pas indispensable pour exprimer la pensée. Suivant un préjugé anti-figuratif, il considère l'écriture chinoise, qui constitue la première écriture réelle, comme supérieure aux hiéroglyphes figuratifs. John Wilkins ${ }^{54}$ (1614-1672) classe les écritures en trois catégories: les écritures courantes, composées d'alphabets, les écritures " pour le secret » et les écritures « pour la brièveté ». Il s'intéresse aux hiéroglyphes et introduit la notion de progrès, considérant l'alphabet comme le résultat d'une évolution. Cependant, les lettres hébraïques demeurent une révélation divine extérieure à cette évolution. Nicolas Fréret (1688-1749) s'intéresse à l'ancienneté comparée de la Chine et de l'Égypte et aux systèmes d'écritures qu'il sépare en deux catégories : phonétiques et non phonétiques. De plus, il estime qu'il n'existe pas de preuve d'un lien entre les langues alphabétiques. Il montre que les caractères chinois ne constituent pas une écriture philosophique et qu'il est impropre de leur donner le nom de hiéroglyphes. Il suppose que les hiéroglyphes d'Égypte sont représentatifs d'idées - il ne parle jamais de valeur phonétique - et que cette écriture est encore absolument inintelligible, balayant ainsi les idées de Kircher. Il n'envisage aucun déchiffrement possible et donc aucune utilisation de la langue copte bien qu'il pense qu'elle ait un lien avec la langue parlée par les Anciens Égyptiens.

\section{Warburton}

Enfin, en 1742, le prêtre anglais William Warburton (1698-1779) édite The divine legation of Moses demonstrated on the principles of a religious deist, from the omission of the doctrine of a future state of reward and punishment in the Jewish dispensation.

En 1744, Léonard des Malpeines publie la traduction française des chapitres concernant les hiéroglyphes en les enrichissant de notes et de planches, sous le titre : Essai sur les hiéroglyphes des Égyptiens: où l'on voit l'origine et le progrès du langage et de l'écriture, l'antiquité des sciences en Égypte, et l'origine du culte des animaux ${ }^{55}$. L'accélération extrême des échanges d'informations en 250 ans est remarquable. En effet, il a fallu 86 ans entre la découverte du manuscrit d'Horapollon et sa publication, 14 ans pour que l'ouvrage de José de Acosta soit traduit en français et en anglais, et seulement 2 ans pour que la traduction française de l'ouvrage de Warburton soit éditée avec commentaires, développements, conclusions...

31 Le but de Warburton n'est pas de déchiffrer les hiéroglyphes mais de renouveler les études bibliques. À cette fin, il propose une histoire théorique de l'écriture en prenant en compte la forme des signes et la fonction des systèmes. Il s'intéresse à la place des hiéroglyphes plutôt qu'à la question chinoise. Il considère que l'évolution des écritures suit quatre étapes. Il suppose que tous les signes sont originellement figuratifs : des «peintures» ou des «représentations naturelles des choses», comme les figures mexicaines. Dans un deuxième temps, la figure cesse d'adhérer étroitement à l'objet et constitue une écriture d'idées, de significations analogiques ou métaphoriques, comme les hiéroglyphes égyptiens. Dans un troisième temps, l'écriture devient courante en simplifiant les signes hiéroglyphiques. Il donne comme exemple le chinois, mais s'intéresse surtout au hiératique, car il suppose, sans pouvoir le vérifier, que cette forme dérive des hiéroglyphes pour en faire une écriture courante. Sa dernière étape est celle de l'écriture de lettres, c'est-à-dire de signes phonétiques. Il propose que ce 
type d'écriture, d'abord secret, devienne commun, alors que les hiéroglyphes, qui n'étaient à l'origine ni secrets, ni mystérieux, car d'usage commun, deviennent secrets. En dernier lieu, il est le premier à annoncer que l'alphabet hébreu n'est pas un don de Dieu, mais résulte de cette évolution, transgressant de fait un interdit religieux considérable. Pour Warburton, même si les écritures sont diverses, elles sont toutes liées. Il suppose à raison qu'on employait simultanément dans l'Égypte ancienne des hiéroglyphes de divers niveaux d'évolution.

\section{Barthélemy}

Dès réception de l'œuvre de Warburton, Jean-Jacques Barthélemy (1717-1795), garde du cabinet des médailles de Louis XV, comprend quel parti en tirer. Puisque les écritures dérivent les unes des autres, il faut les déchiffrer en remontant le temps depuis la plus récente. De plus, il faut se méfier des auteurs antiques :

Ces questions ne pourront jamais être éclaircies par les témoignages des auteurs grecs et latins... C'est aux monuments qu'on doit recourir. Quand ils parleront clairement, il faudra bien que les anciens auteurs s'accordent avec eux ${ }^{56}$.

Déchiffrer une écriture nécessite une inscription bilingue. Il faut compter les signes et les comparer avec ceux d'une seule écriture qui lui ressemble; sur ce point il hérite de la pensée de Vigenère. Il faut confronter la langue à déchiffrer à une autre qu'on connaît, de même famille, et une seule. Dans l'inscription bilingue, il faut utiliser les noms propres pour retrouver la valeur des lettres qui les composent. Il faut qu'une lettre exprime toujours la même valeur. Il faut donc une valeur par lettre, une lettre par valeur et ne pas en changer selon ses désirs. Cela fait de lui l'inventeur de la méthode du déchiffrement.

Grâce aux copies exactes de James Dawkins et Robert Wood ${ }^{57}$ qui montrent de courtes inscriptions bilingues, il déchiffre d'abord le palmyrénien et présente sa découverte à l'Académie le 12 février $1754^{58}$. Il fait l'état de la question, énonce la méthode qu'il a mise au point et montre son application couronnée de succès à la question palmyrénienne. Par la suite, il déchiffre le phénicien en utilisant les moulages en plâtre de deux monuments maltais que lui avait envoyés son ami le comte Anne-ClaudePhilippe de Caylus (1692-1765). Il présente ses résultats à l'Académie le 12 avril $1758^{59}$ (fig. 8). Il lit d'abord le texte grec $\triangle$ IONY $\Sigma I O \Sigma$ KAI $\Sigma A P A \Pi I \Omega N$ OI $\Sigma A P A \Pi I \Omega N O \Sigma$ TYPIOI HРАK $\Lambda$ EI APXНГETEI «Dionysios et Sarapion fils de Sarapion, Tyriens, à Héraklès le fondateur ». Il est plus court que le texte phénicien, ce qui constitue une difficulté. Barthélemy constate que les noms propres du texte grec sont une traduction des noms phéniciens et non une simple transposition phonétique. Il doit donc comprendre ce que signifient ces noms propres théophores en trouvant les équivalents phéniciens des divinités évoquées, Dionysos et Héraclès. Puisque Barthélemy dirige le cabinet des médailles de Louis XV, il fait entrer dans les collections un grand nombre de monnaies phéniciennes pour pouvoir y compter les signes. Il conclut que cette écriture est constituée du même nombre de lettres que l'alphabet hébreu qui n'utilise que des consonnes et des consonnes faibles (ce que nous appelons les matres lectionis). Il considère qu'il s'agit d'une langue proche de l'hébreu. En conséquence, pour trouver la

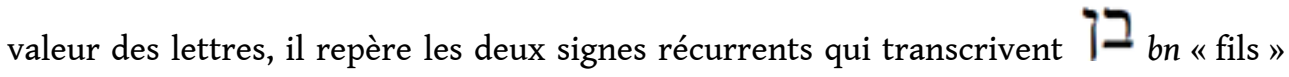
entre les noms propres qu'il repère. En comprenant que Héraklès correspond à Melqart et Dionysos à Osiris ${ }^{60}$, il déduit les valeurs des signes qui les composent. Ainsi, il 
obtient : « Abdassar \& Asseremor, fils d'Asseremor, fils d'Abdassar, avons fait ce vœu à notre seigneur Melcrat, divinité tutélaire de Tyr. Puisse-t-il les bénir après les avoir égarés ». Aujourd'hui nous lisons : l'dnn l Mlqrt b'l șr 'š ndr 'bdk 'bd-'sr w 'hy 'sr-šmr šn bn 'sr-šmr bn 'bd-'sr k šm' qlm ybrkm « Pour notre seigneur, pour Melqart seigneur de Tyr, ce qu'a voué ton serviteur 'Abdosir et son frère Osirchamar, tous deux fils de Osirchamar fils de 'Abdosir, car il a entendu leur voix, qu'il les bénisse.» Nous constatons que seule la fin de la dernière proposition de sa traduction est fausse. Deux siècles et demi plus tard, il obtiendrait encore une excellente note à l'examen de langues sémitiques à l'École du Louvre.

Fig. 8

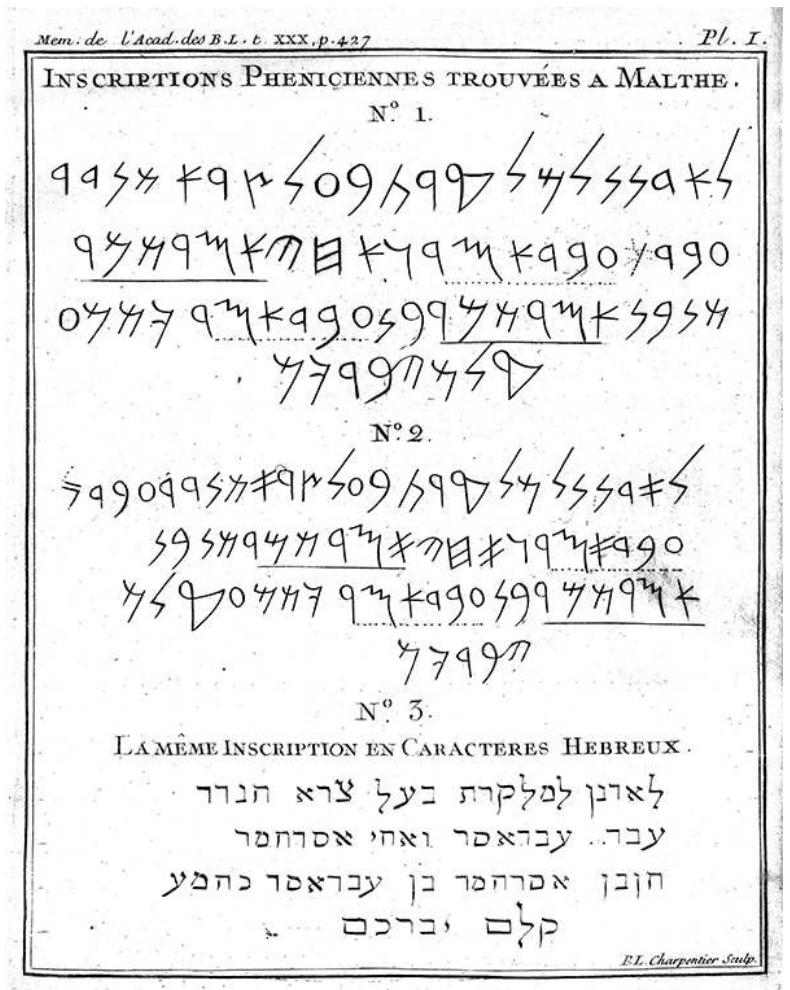

Jean-Jacques Barthélemy, « Réflexions sur quelques monumens phéniciens et sur les alphabets qui en résultent », Mémoires de l'Académie des Belles Lettres, t. XXX, 1758, pl. I

L'une des deux bases de marbre se trouve aujourd'hui à la Bibliothèque publique de La Valette; l'autre fut offerte en 1780 au roi Louis XVI, en hommage pour le déchiffrement de l'abbé Barthélémy, par le chevalier de Rohan, commandeur des chevaliers de Malte, et le roi ordonna de la déposer au Palais Mazarin. L'Académie des Inscriptions et Belles-Lettres, quand elle fut installée dans ledit Palais, en devint la gardienne ; elle l'offrit ultérieurement au Musée du Louvre ${ }^{61}$.

De nos jours, le cippe de Barthélemy AO 4818 est présenté dans la salle 18 b, à côté de la fenêtre. Il y a quelques années, l'inscription se trouvait à contre-jour, ce n'est heureusement plus le cas aujourd'hui. Cependant, en raison de son importance dans l'histoire du déchiffrement, ce monument mériterait d'être présenté au Louvre à une place d'honneur, à côté d'une figure de Barthélemy accompagnée de quelques explications correspondant à la reconnaissance qui devrait être la nôtre vis-à-vis de son œuvre. 
Deux ans plus tard, en cherchant l'obélisque de Rome traduit par Hermapion dans l'espoir d'obtenir ainsi l'inscription bilingue nécessaire au déchiffrement, Barthélemy

déduit que les ovales $\longrightarrow$ qui entourent certains groupes de hiéroglyphes renferment des noms royaux ${ }^{62}$. La compréhension du sens des cartouches fait de Barthélemy le premier homme à déchiffrer un hiéroglyphe! Il enseigne que lorsqu'un texte bilingue aura été trouvé, il faudra en commencer le déchiffrement par ces noms propres. N'ayant pas trouvé l'obélisque traduit par Hermapion, il abandonne la partie, non sans avoir annoncé que les hiéroglyphes constituent une véritable écriture qui transcrit une langue et que cette langue est le copte.

On pourrait croire, dès lors, les propositions farfelues sur les hiéroglyphes évacuées pour toujours. Que nenni ! Par exemple, si Joseph de Guignes (1721-1800) développe en 1785 l'hypothèse de Barthélemy concernant les cartouches royaux, il fait aussi considérablement reculer la science égyptologique à naître du fait de son hypothèse, énoncée le 14 novembre 1758 à l'Académie ${ }^{63}$, au sujet du lien de parenté entre écriture chinoise et écriture hiéroglyphique - supposition qui remonte au moins à Blaise de Vigenère. Il développe la thèse selon laquelle ces ressemblances seraient le résultat d'une colonisation de la Chine par les Égyptiens ${ }^{64}$ à l'époque du grand conquérant Sésostris. L'idée peut faire sourire, mais on est moins enclin à la juger trop sévèrement lorsqu'on songe que l'a priori presqu'aussi absurde et naif d'une colonisation de l'Égypte par les Sumériens est parfois encore soutenu, y compris par des médias qu'on pourrait croire dignes de confiance. Pourtant, nous pouvons remercier Guignes pour cette théorie, car, bien malgré lui, il a permis à Champollion de déchiffrer les hiéroglyphes. En effet, se fondant sur cette colonisation, Guignes suppose que l'écriture égyptienne comporte des "clefs " comparables à celles de l'écriture chinoise. "Les Égyptiens ont donné aux Chinois tout leur système d'écriture, car les signes de ce dernier sont en partie hiéroglyphiques et en partie alphabétiques ${ }^{65}$. " Sa publication pose des questions qui seront fondamentales pour les réflexions de Champollion : «Un même hiéroglyphe pouvait-il avoir des sens différents, selon qu'il était diversement employé dans la suite du discours comme il arrive aux caractères chinois ${ }^{66}$ ? » De plus, bien que ses rapprochements soient dénués de sens, Guignes développe une remarque fondamentale concernant la relation entre le nom des lettres et la gestation de l'alphabet à partir de signes figuratifs qui ne prendra tout son sens qu'en 1916 lorsque Gardiner déchiffrera l'écriture protosinaïtique découverte onze ans plus tôt par Flinders Petrie au Serabit el-Khâdim dans le Sinaï ${ }^{67}$.

\section{La pierre de Rosette !}

En 1798, le gouvernement français décide d'envoyer une expédition en Égypte pour couper la route des Indes afin d'affaiblir l'Angleterre. Conformément aux idéaux de la Révolution et du siècle des Lumières, des savants sont envoyés pour décrire tous les aspects possibles de l'Égypte ancienne et contemporaine et rapporter des documents de toutes sortes. Les monuments pharaoniques ainsi que leurs hiéroglyphes sont, bien entendu, au centre des préoccupations. Afin de divulguer les idéaux révolutionnaires et d'éclairer les populations, on emporte une arme décisive encore inconnue en Égypte: l'imprimerie. Dans l'Empire ottoman, par suite des édits de Bajazed II en 1485 et de son fils Sélim $I^{\text {er }}$ en 1515 , cet usage est interdit pour les textes en caractères arabes ${ }^{68}$. De ce fait, c'est seulement au début $d u x_{\text {XIII }}{ }^{e}$ siècle qu'y sont édités les premiers livres 
imprimés en arabe, à Alep, puis à Constantinople ${ }^{69}$. La divulgation des découvertes scientifiques de l'expédition française est donc assurée par la première imprimerie d'Égypte, fondée à Alexandrie en juillet 1798, "l'Imprimerie orientale », qui édite en caractères latins, grecs et arabes. En septembre 1798, cette « Imprimerie orientale » est déplacée au Caire, renommée «Imprimerie nationale » et placée sous la responsabilité de Jean-Joseph Marcel. Joseph Marc Emmanuel Aurel dirige une autre imprimerie, indépendante, qu'il cède avant de rentrer en France en 1800. Elles cessent leurs activités en 1801 à la suite de la défaite française. Quoi qu'il en soit, l'objectif scientifique transforme le désastre militaire que l'on sait en succès moral pour la Révolution française et pour le futur empereur.

Le 19 juillet 1799, six jours avant la victoire de Bonaparte sur les Ottomans à Aboukir, le lieutenant du génie Pierre François Xavier Bouchard, lors du renforcement du vieux fort délabré de la ville côtière de Rachid, baptisé Fort Julien par les membres de l'expédition de Bonaparte, découvre une stèle trilingue que nous appelons «pierre de Rosette $^{70} »$ (fig. 9). De bas en haut, elle comporte 54 lignes en grec, tronquées à l'angle inférieur droit. La partie la mieux conservée est inscrite de 32 lignes en démotique, dont il ne manque que le début des 14 premières. La partie supérieure, hiéroglyphique, est la plus incomplète : il n'en reste que 14 lignes dont aucune n'est entière.

Fig. 9

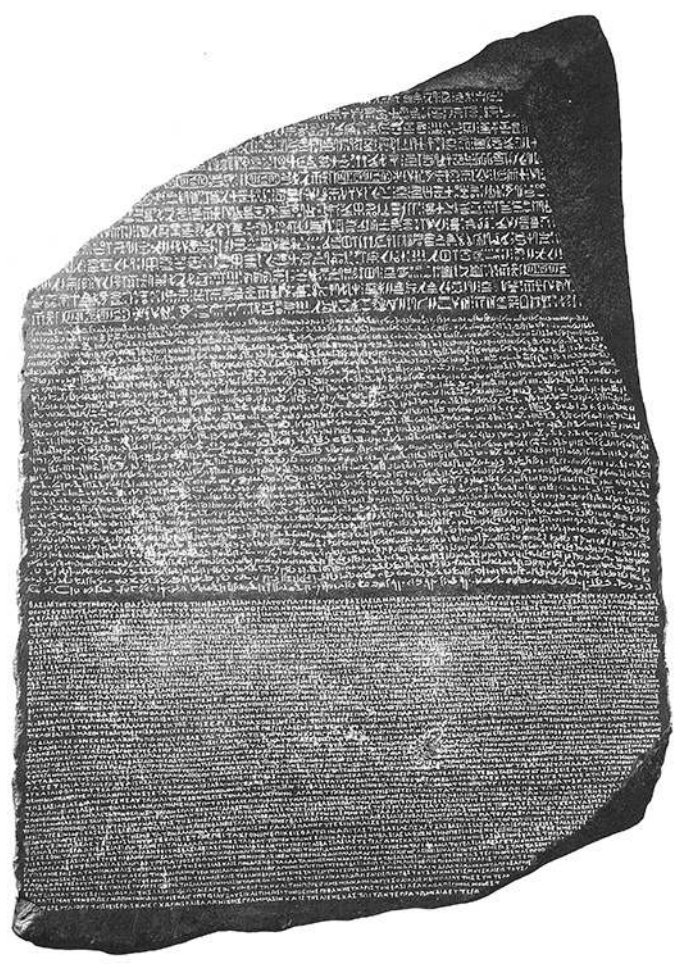

Pierre de Rosette - Londres, British Museum

(C) Institut Khéops

Le 29 juillet 1799, lors de la $31^{\mathrm{e}}$ séance de l'Institut d'Égypte, on lit la lettre dans laquelle l'ingénieur Michel-Ange Lancret, membre de l'Institut, informe que le citoyen Bouchard a découvert une pierre noire trilingue « dont l'examen peut offrir beaucoup d'intérêt ». Il s'agit d'un décret de Ptolémée V Épiphane datant du 27 mars 196 avant 
J.-C. Pour la reproduire exactement, Jean-Joseph Marcel, assisté d'Antoine Galland, invente un nouveau procédé. Il s'agit de "l'autographie " qui permet d'obtenir une impression inversée du texte composé de signes en creux qui apparaissent en blanc sur fond noir. Nicolas Conté utilise le procédé de la « chalcographie » qui permet d'obtenir une impression du texte où les signes en creux apparaissent inversés et en noir sur fond blanc. Adrien Raffeneau-Delille effectue un moulage à base de souffre. Au printemps 1800, le général Dugua emporte en France une épreuve de Jean-Joseph Marcel et une épreuve de Nicolas Conté pour les confier à l'Institut national de Paris. La publication de la Description de l'Égypte utilisera la reproduction au souffre de Adrien Raffeneau-Delille.

Lorsque les Français sont battus par les Ottomans et les Anglais en 1801, ces derniers exigent la plupart des objets archéologiques, en particulier la pierre de Rosette. C'est actuellement le premier monument que voit le visiteur en entrant dans le département des Antiquités égyptiennes du British Museum. Sur sa tranche, est gravé : «Captured in Egypt by the British Army in 1801 » et sur l'autre côté : «Presented by King George III ». Cet objet est, avant tout, le témoin générique des terribles guerres franco-anglaises, que les Anglais méritent de conserver avec une fierté légitime.

Quoi qu'il en soit, l'ultime bataille contre le secret des hiéroglyphes peut enfin être engagée. Barthélemy est mort, mais l'ombre du maître plane au-dessus des savants qui, obéissant à sa méthode, commencent par l'écriture la plus récente : le démotique.

41 Le professeur d'arabe et de persan au Collège de France Antoine-Isaac Silvestre de Sacy (1758-1838), référence des orientalistes de son temps, joue en quelque sorte le rôle d'arbitre auprès de ceux qui tentent de déchiffrer la pierre de Rosette. Il ouvre les hostilités en obéissant aux préceptes de Barthélemy et repère la position de cinq cartouches royaux dans le texte égyptien, ce qu'il publie en $1802^{71}$.

Parmi les étudiants de Sylvestre de Sacy, se trouve un Suédois, Johan David Åkerblad (1763-1819) qui maîtrise plus de vingt langues antiques et contemporaines. Appliquant les préceptes de Barthélemy, il s'attaque au démotique, l'écriture la plus récente, mais la plus difficile à lire. En utilisant les découvertes de Sylvestre de Sacy, il étudie dans le texte démotique les noms propres qu'on trouve dans le texte grec et compare la langue démotique à la langue copte. Ainsi, en seulement deux mois, il réussit à déchiffrer une partie de l'inscription démotique, à savoir, tous les noms propres et un certain nombre de mots de vocabulaire. Il publie ses résultats en $1802^{72}$, ce qui fait de lui le premier homme moderne à déchiffrer de l'égyptien pharaonique. Malheureusement, il reste persuadé que les signes démotiques et hiéroglyphiques expriment des sons.

Entre en scène Thomas Young (1773-1839). Dans la tradition des déchiffreurs, c'est un adepte des sciences "dures ", mathématiques, physique, médecine... Il est avant tout médecin, mais compte à son actif des découvertes fondamentales en physique, qui portent son nom. Comme il se doit, il connaît une quinzaine de langues anciennes et modernes. Ce qu'on sait moins, c'est qu'il est l'un des inventeurs de la notion de langues indo-européennes mise au point grâce à la comparaison de centaines d'idiomes différents en 1813. Une telle personnalité ne pouvait pas ne pas s'intéresser à la pierre de Rosette. Suivant Barthélemy, il s'attaque au démotique et aux cartouches royaux. Åkerblad transmet le résultat de ses recherches sur le démotique à Young qui les améliore immédiatement. Il étudie, en particulier, la relation entre la forme des signes démotiques, hiératiques et hiéroglyphiques. Il propose la traduction d'un peu plus de deux cents mots différents. Près de la moitié sont justes, ce qui est considérable. 
Cependant, il n'a pas compris le système hiéroglyphique. En 1814, il montre ses résultats à Sylvestre de Sacy et obtient son soutien.

\section{Les frères Champollion}

Avant 1820, contrairement à une idée reçue, Jean-François Champollion, dit le Jeune (1790-1832), ne consacre pas tout son temps au déchiffrement des hiéroglyphes, car il est occupé par d'autres activités : politique, enseignement ${ }^{73} .$. Quoi qu'il en soit au cours de cette période de formation, il étudie toutes les langues possibles, anciennes et modernes, dont, bien entendu le copte - car dès 1804, il prend connaissance des publications de Guignes et de Barthélemy concernant le lien entre la langue copte et les hiéroglyphes $^{74}$ - en utilisant, entre autres, les livres de la bibliothèque de son frère aîné Jacques-Joseph Champollion-Figeac (1778-1867), qui contient « par principe, tout ce à quoi on ne comprend rien (...) ainsi hébreu, syriaque, sanscrit, tartare, chinois, persan et surtout les langues antiques ${ }^{75} »$. Dans un premier temps, il émet des théories relativement fantaisistes et se perd quelque peu en partant dans toutes les directions, proposant même une origine égyptienne aux Étrusques ou à l'écriture chinoise. Heureusement, son frère trie le bon grain de l'ivraie, le conseille et le ramène régulièrement dans le droit chemin. Dès 1811, à cause du nombre de signes hiéroglyphiques de la pierre de Rosette, il soupçonne que cette écriture soit composée de «signes alphabétiques » - c'est à dire des phonogrammes qui expriment un son - et « d'imitations d'objets naturels » - c'est à dire d'idéogrammes qui expriment une idée. Avant 1822, il change régulièrement d'opinion sur ce point. Les premières réactions de Silvestre de Sacy aux travaux de Champollion sont négatives. Ainsi, en 1815, il écrit à Young :

...Si j'ai un conseil à vous donner, c'est de ne pas trop communiquer vos découvertes à M. Champollion. Il se pourrait faire qu'il prétendît ensuite à la priorité. Il cherche en plusieurs endroits de son ouvrage à faire croire qu'il a découvert beaucoup de mots de l'inscription égyptienne de Rosette. J'ai bien peur que ce ne soit là que du charlatanisme. J'ajoute même que j'ai de fortes raisons de le penser.

On est loin de l'hagiographie présentant les savants français et anglais de l'époque comme des adversaires impitoyables, à la manière de deux équipes de football soutenues par des hooligans ultranationalistes... Il faut comprendre Sylvestre de Sacy : le moins que l'on puisse dire, c'est que les propositions de Champollion à ses débuts sont embrumées et souvent confuses. Quoi qu'il en soit, Sylvestre de Sacy changera d'avis dès que Champollion apportera des éléments tangibles concernant ses théories.

\section{Les prémices}

Une lettre que Champollion adresse à François Arthaud le 18 septembre $1820^{76}$ montre qu'il a obtenu dès cette époque quelques résultats notables permettant de comprendre la nature de ses découvertes postérieures. Il sait déjà que le signe $\quad$ signifie «dieu » dans 16 qu'il traduit « Dieu gracieux ${ }^{77}$ ». Grâce à la pierre de Rosette, et par analogie avec un principe de l'écriture chinoise, il a trouvé que le pluriel peut être obtenu en 
triplant le signe ou en lui adjoignant trois traits :

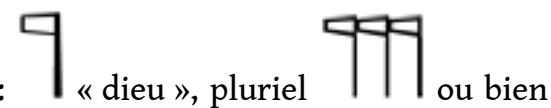

पi

«dieux " ${ }^{78}$. "Mais une divinité spécialement déterminée y est représentée symboliquement par un corps humain accroupi à l'Égyptienne et surmonté de la tête de l'animal emblème vivant et terrestre de cette même divinité ${ }^{79}$ ». Il pense que les hiéroglyphes I ff sens du mot véhiculé par le signe $\boldsymbol{f}^{\prime}$, ce qui sera capital deux ans plus tard. En revanche, à cette date, il n'a toujours pas compris que le groupe $\sqrt{\text { correspond au }}$ nom d'Osiris ${ }^{81}$.

À la suite des troubles de 1821 survenus à Grenoble, la perte de ses emplois l'oblige à monter à Paris et à consacrer enfin tout son temps au déchiffrement de l'égyptien avec les résultats que l'on sait. Concernant le texte démotique de la pierre de Rosette, Champollion présente devant l'Académie, le 26 juillet 1822, un mémoire resté inédit, puis un nouveau mémoire, les 23,30 août et 20 septembre $1822^{82}$.

\section{Un alphabet pour transcrire les noms des princes grecs et romains}

Comme son sous-titre l'indique ${ }^{83}$, la Lettre à Monsieur Dacier, publiée en 1822, traite principalement des signes hiéroglyphiques utilisés pour transposer phonétiquement les noms des souverains gréco-romains de l'Égypte.

Il s'agit de la série des hiéroglyphes qui, faisant exception à la nature générale des signes de cette écriture, étaient doués de la faculté d'exprimer des sons des mots, et ont servi à inscrire sur les monuments publics de l'Égypte, les titres, les noms et les surnoms des souverains grecs et romains qui la gouvernèrent successivement ${ }^{84}$.

En premier lieu, Champollion annonce «croire être parvenu, après dix années de recherches assidues, à réunir des données presque complètes sur la théorie générale de ces deux espèces d'écriture, sur l'origine, la nature, la forme et le nombre de leurs signes, les règles de leurs combinaisons au moyen de ceux de ces signes qui remplissent des fonctions purement logiques ou grammaticales ${ }^{85} »$. En fait, cette annonce est présomptueuse, car il a accès à un nombre insuffisant d'inscriptions égyptiennes pour une étude aussi complète.

La pierre de Rosette a suffi pour déchiffrer le démotique et Champollion rappelle à BonJoseph Dacier (1742-1833) ce qu'il doit sur ce point à " votre illustre confrère Silvestre de Sacy, et successivement à (...) feu Akerblad et M. le docteur Young ». Champollion considère que l'écriture démotique transcrit phonétiquement les noms étrangers « au moyen de signes plutôt syllabiques qu'alphabétiques ${ }^{86}$ ", il parle même de "syllabaire démotique ${ }^{87}$ ». Il prête à "syllabique » un sens différent de ce que nous entendons actuellement par ce terme. Il s'explique clairement dans la légende de la planche IV.

Tous les signes hiéroglyphiques ou démotiques qui répondent aux consonnes de l'alphabet grec, prennent une valeur véritablement syllabique, lorsqu'ils sont combinés entr'eux sans mélange d'autres signes de voyelle. C'est ainsi, par exemple, que le nom phonétique de Bérénice renfermé dans le cartouche $\mathrm{n}^{\circ} 32$, devrait se lire et se transcrire $\mathrm{B} \varepsilon-\mathrm{P} \varepsilon-\mathrm{NI}-\mathrm{K} \varepsilon-\Sigma$. Le signe phonétique des articulations $\mathrm{B}, \mathrm{P}, \mathrm{N}$, ainsi que ceux des autres consonnes $\Gamma, \Delta, \Lambda, M, N, \Pi, \Sigma, T$, etc., représentent, dans ces 
occasions très-ordinaires, les syllabes $\mathrm{B} \varepsilon, \Gamma \varepsilon, \Delta \varepsilon, \mathrm{K} \varepsilon, \Lambda \varepsilon, \mathrm{M} \varepsilon, \mathrm{N} \varepsilon, \Pi \varepsilon, \mathrm{P} \varepsilon, \Sigma \varepsilon$, Te, etc. On a dû remarquer en effet que presque toujours, les Égyptiens n'écrivaient dans les noms phonétiques, que les seules voyelles longues ainsi que les diphtongues. Les voyelles brèves comprises dans le corps des mots, ne sont presque jamais exprimées, parce que le signe de la consonne les emporte en lui-même et devient, par cela même, un caractère syllabique.

Il ajoute : «Les signes des voyelles A H E I s'emploient assez indifféremment l'un pour l'autre.» Il utilise donc "syllabique» pour décrire un système consonantique comparable aux écritures sémitiques du type phénicien, hébreu ou arabe ${ }^{88}$. Les voyelles longues et les diphtongues dont il parle sont ce que nous appelons les «matres lectionis ».

En raison de l'état de la partie supérieure de la pierre de Rosette, ce qui subsiste du texte hiéroglyphique ne conserve que le nom de Ptolémée, inscrit cinq fois. Pour pallier ce déficit, Champollion a recours à un expédient astucieux. Il utilise le texte du socle de l'obélisque de Philae "récemment transporté à Londres », copié par Cailliaud ${ }^{89}$, qui cite en grec le "roi Ptolémée ", "Cléopâtre sa sœur » et "Cléopâtre sa femme », comme bilingue pour retrouver les noms royaux des cartouches hiéroglyphiques inscrits sur l'obélisque ${ }^{90}$. Il y reconnaît Ptolémée "conçu dans les mêmes signes que dans l'Inscription de Rosette également renfermé dans un cartouche ». Il considère que le second cartouche de l'obélisque «doit contenir nécessairement le nom propre d'une femme, d'une reine Lagide, puisque ce cartouche est terminé par les signes hiéroglyphiques du genre féminin, signes qui terminent aussi les noms propres hiéroglyphiques de toutes les déesses égyptiennes sans exception ». Il s'agit de la lettre

$t \bigcirc$, marqueur grammatical du féminin en égyptien, suivie de l'œuf $\bigcirc$, déterminatif du féminin. Il lit les deux signes comme un tout ${ }^{91}$. En toute logique, il conclut que ce ne peut être "que celui d'une Cléopâtre ${ }^{92}$ ». Il compare la position des signes hiéroglyphiques correspondant aux lettres semblables de la version grecque de ces noms, ce qui lui permet de "constater leur nature entièrement phonétique " et d'obtenir les valeurs d'une liste de «douze signes répondant à onze consonnes et voyelles ou diphtongues de l'alphabet grec: $\mathrm{A}, \mathrm{AI}, \mathrm{E}, \mathrm{K}, \Lambda, \mathrm{M}, \mathrm{O}, \Pi, \mathrm{P}, \Sigma, \mathrm{T}$ », en tenant compte de l'homophonie de certains hiéroglyphes ${ }^{93}$. Il peut alors les utiliser pour déchiffrer d'autres noms propres et cherche dans ce but tous les cartouches de la période gréco-romaine qu'il peut trouver sur les monuments à sa disposition : le temple de Philæ ${ }^{94}$, le grand temple d'Ombos ${ }^{95}$, le grand temple d'Edfou ${ }^{96}$, les temples de " $\operatorname{Karnac}^{97} »$, le temple de Médinet Habou à Thèbes ${ }^{98}$, le monolithe de Qous ${ }^{99}$, les monuments de Dendéra ${ }^{100}$, la pierre de Rosette ${ }^{101}$; à Rome, l'obélisque Pamphile ${ }^{102}$, l'obélisque Barbérini ${ }^{103}$... Il obtient ainsi les noms hiéroglyphiques d'un nombre conséquent de rois macédoniens, dont il donne la translitération à l'aide de lettres grecques : Alexandre ${ }^{104}$ (A $\Lambda K \Sigma A N T P \Sigma$, A $\Lambda K \Sigma N T P E \Sigma$, APK $\left.\Sigma N T P \Sigma\right)$ ), Ptolémée ${ }^{105}$ (ПTO $\Lambda \mathrm{MH} \Sigma$,

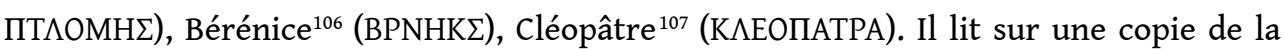
paroi extérieure du temple d'Hathor de Dendéra le nom de Ptolémée-Césarion en

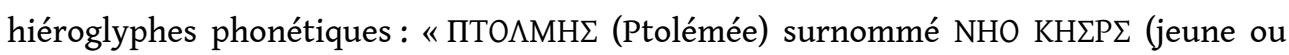
nouveau César) ${ }^{108}$ » et « ПTO $\Lambda$ MH $\Sigma$ KH $\Sigma \Lambda \Sigma$ (pour KH $\Sigma$ P $\Sigma$ ) Ptolémée-Cæsar ${ }^{109}$ ».

51 Il constate que le nom de ces souverains macédoniens « est presque toujours précédé d'un autre cartouche qui contient » leurs «surnoms particuliers ${ }^{110}$ » et que leur nom dans le second cartouche est souvent « accompagné des titres idéographiques toujours vivant, chéri de Phtah ; toujours vivant, chéri d'Isis ; toujours vivant, chéri de Phtah et 
d'Isis », « Dieu sauveur », « Dieu Évergète », « Dieu Épiphane », « Dieu Adelphe », « les dieux évergètes chéris ", "les dieux évergètes chéris d'Isis ", "sa femme ${ }^{111}$ ", "sa sœur ${ }^{112} »$. Il a donc approfondi ses découvertes exprimées dans sa lettre de 1820.

Il déchiffre aussi « les titres, les noms et jusqu'aux surnoms des empereurs romains, énoncés en langue grecque et écrits avec ces mêmes hiéroglyphes phonétiques ${ }^{113}$ ". Pour ce faire, il tire profit des légendes grecques des monnaies de l'époque ${ }^{114}$. Il comprend qu'il peut les employer comme bilingues car il constate que leurs tournures correspondent à celles qui accompagnent les noms des empereurs romains dans les cartouches, ce qui permet de les identifier. Se servir des médailles pour déchiffrer des inscriptions monumentales place Champollion dans la droite ligne de Barthélemy. Par

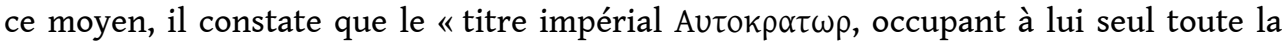
capacité d'un cartouche, ou bien encore suivi des titres idéographiques toujours vivant, orthographié АОТОКРТР, АОТКРТОР, АОТАКРТР, et même АОТОК $\Lambda$ T $\Lambda^{115}$ ", ou "AOTKPTP116", est presque toujours accolé " ou mis en rapport avec un second cartouche contenant (...) les noms propres des empereurs». Ainsi, il reconnaît Auguste $^{117}$, Tibère ${ }^{118}$, Domitien ${ }^{119}$, Vespasien ${ }^{120}$, Trajan $^{121}$, Hadrien $^{122}$ et son épouse Sabine ${ }^{123}$, ainsi qu'Antonin le Pieux ${ }^{124}$.

\section{La découverte de la clef du déchiffrement}

Dans la Lettre à Monsieur Dacier, Champollion annonce un «mémoire sur les "hiéroglyphes purs" " à venir ${ }^{125}$. Cependant, il publie ce document en avant-première pour offrir aux savants qui travaillent sur la question des hiéroglyphes « une courte mais importante série de faits nouveaux (...) qui leur épargnera (...) peut-être aussi de graves erreurs (...) ». Il affirme que l' « alphabet » utilisé pour écrire les noms grecs et romains a été inventé bien avant, pour écrire des sons entrant dans la composition des noms de pharaons égyptiens ${ }^{126}$. Il a compris ce point capital grâce à des cartouches royaux copiés entre le 21 janvier et le 8 février 1819 à Abou Simbel par l'architecte Jean-Nicolas Huyot ${ }^{127}$ (1780-1840) qui les lui a envoyés. Champollion y reconnaît le signe

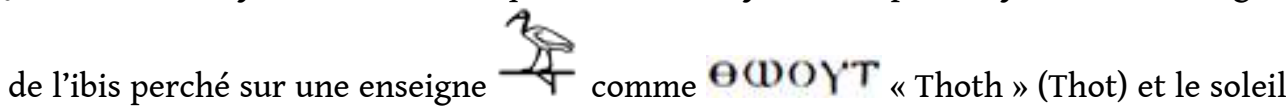
$\odot_{\text {comme }} \Pi \mathrm{PH}$ ou «Rha» (Rê). En 1820, il pensait que le groupe I f ft formé des signes $\odot$, I et if

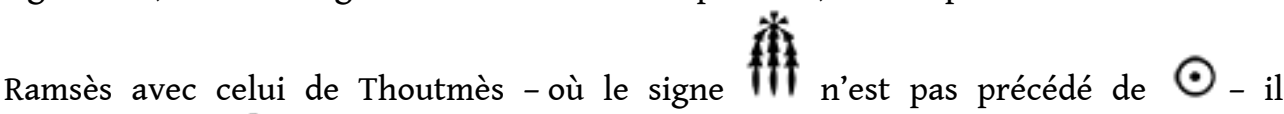
comprend que 数 seul exprime l'idée de naissance et que $\odot$ signifie « soleil ", "Rê ». Il repère aussi dans les deux cartouches le $\|_{s \text { de Ptolemaios derrière le signe }}$ 甜 C'est à cette occasion que Champollion comprend que son "alphabet " n'est pas seulement destiné à transposer phonétiquement les noms gréco-romains, mais que son usage est beaucoup plus ancien. Il vient de découvrir que ces signes phonétiques aident à la lecture des idéogrammes dans les noms des rois égyptiens. Ainsi, il a la preuve que leur création n'est pas causée par le besoin d'écrire des noms étrangers pensés dans 
une autre langue et transposés depuis un autre système d'écriture. De plus, ce $\|_{s \text { lui }}$ prouve que les hiéroglyphes expriment du copte. En effet, cette lettre aide à comprendre le sens de son idéogramme 数 "naître ", c'est-à-dire MlC $\mathrm{E}$ en copte. Le 14 septembre 1822, il lit donc PHMCC «Rhamsès " (Râmsès) et O(DOYTMC (Thoutmès / Thoumosis) en comprenant la signification de ces noms qu'il pense en copte. La découverte de Champollion n'est pas à proprement parler la valeur phonétique du signe f $_{\text {t }}$ ms qu'il considère comme un idéogramme, mais l'emploi des compléments phonétiques unilitères destinés à éclairer le lecteur dès les époques anciennes pour exprimer des mots de la langue égyptienne pharaonique.

Pourtant, dans la Lettre à Monsieur Dacier, Champollion se contredit à plusieurs reprises ; ainsi, lorsqu'il affirme que «la plupart d'entre ces monuments portant à la fois des noms pharaoniques, des noms grecs et des noms romains, et les premiers, caractérisés par le petit nombre de leurs signes, résistant constamment à toute tentative pour $\mathrm{y}$ appliquer avec succès l'alphabet que je viens de faire connaître ${ }^{128}$ ». Force est de constater que Champollion a inséré ses dernières découvertes dans un texte rédigé antérieurement qu'il n'a pas eu le temps d'expurger.

En 1824, il rappelle dans l'introduction de son Précis du système hiéroglyphique des Anciens Égyptiens, que la publication de la Lettre à Monsieur Dacier montrait que «les anciens Égyptiens s'étoient servis de signes hiéroglyphiques purs (...) pour représenter les sons des noms de souverains Grecs ou Romains (...) ; et dès cette époque, j'avais acquis l'intime conviction que l'usage de ces mêmes caractères phonétiques, ou exprimant des sons, remontait à un temps antérieur aux dynasties étrangères en Égypte ${ }^{129}$. " Il annonce que le but principal du Précis est de démontrer :

$1 .^{\circ}$ Que mon alphabet hiéroglyphique s'applique aux légendes royales hiéroglyphiques de toutes les époques; $20^{\circ}$ Que la découverte de l'alphabet phonétique des hiéroglyphes est la véritable clef de tout le système hiéroglyphique ; 3..$^{\circ}$ Que les anciens Égyptiens l'employèrent, à toutes les époques, pour représenter alphabétiquement les sons des mots de leur langue parlée; $4 .^{\circ}$ Que toutes les inscriptions hiéroglyphiques sont en très-grande partie, composées de signes purement alphabétiques, et tels que je les ai déterminés (...) cette théorie (...) nous donnera bientôt l'intelligence pleine et entière de tous les textes hiéroglyphiques ${ }^{130}$.

De fait, en annonçant la méthode grâce à laquelle il va déchiffrer l'égyptien hiéroglyphique, il avoue que s'il a effectivement compris l'essentiel de son système, il n'a pas encore totalement abouti. La raison vient du fait qu'il manque d'inscriptions hiéroglyphiques et hiératiques pour pouvoir en vérifier la validité. C'est pourquoi, en 1824, il part à Turin étudier la collection égyptienne. Il ne sera pas déçu. Il y constate la validité de son système. Cependant, ce n'est pas encore suffisant.

C'est son voyage en Égypte de 1828 à 1829 qui, en lui livrant tous ces monuments originaux qui lui faisaient défaut, lui permet de prouver définitivement le bien-fondé de sa découverte. À l'aller, il repère ce qu'il copiera au retour. Son courrier témoigne de ses progrès. En route vers le Sud, il emmagasine les informations sans traduire vraiment les inscriptions. En revanche, au retour, il envoie les traductions de textes entiers. C'est donc seulement en 1829 que nous pouvons considérer qu'il a achevé son processus de déchiffrement du système hiéroglyphique et qu'il lit effectivement 
l'égyptien ancien. Dans son journal de voyage, à la date du 2 octobre 1828, il lit à

Tourah le nom de la reine

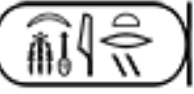

二頖

A2 $\mathrm{MC}$ «Ahmosis ${ }^{132}$ » qui s'écrivent à l'aide du signe

秝

écrit pendant ce voyage d'Égypte, la transcription copte montre que Champollion lit en suivant l'ordre d'écriture des signes sans tenir compte de l'antéposition honorifique :

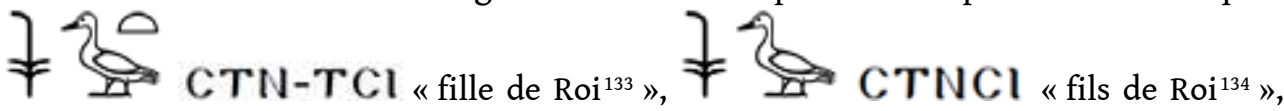

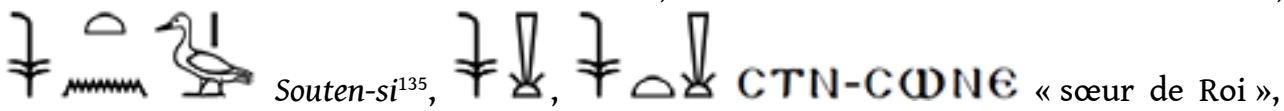

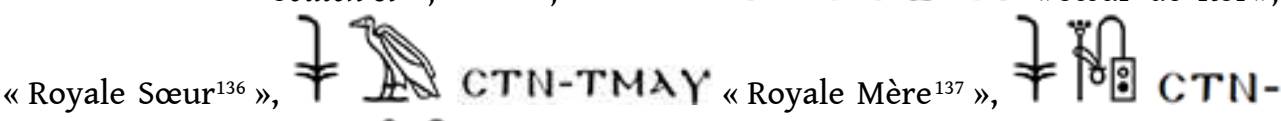
CA2 «scribe royal», de Phtha », 2A $\Theta$ (DP-MAl « Aimé d'Athyr ${ }^{139}$ ».

Ce qui est incroyable, c'est que Champollion n'a pas fait table rase d'Horapollon, mais qu'il réussit à utiliser efficacement le contenu des Hiéroglyphica. On le constate, par exemple, dans sa lettre de réponse au philosophe et diplomate Wilhelm von Humboldt

(1767-1835) du 12 février $1825^{140}$, lorsqu'il analyse le signe du lièvre :

(...) c'est là l'animal désigné sous le nom Grec du lièvre $\Lambda \alpha \gamma \omega \varsigma$ dans le texte d'Horapollon, où on lit que cet animal était le signe de tout ce qui est ouvert; or je le trouve employé à exprimer les idées paraître, être patent dans les textes Égyptiens, et l'analogue des racines OYWN, aperire, ouvrir, enseigner, et COYCN, CWOYN, connaitre et ouvrir, montrer, est trop marquée dans les restes de la langue Égyptienne pour ne point reconnaître l'identité de l'animal appelé lièvre par Horapollon avec celui qui est le signe des idées : être patent, être ouvert, être permis, dans les textes hiéroglyphiques.

Dans sa lettre écrite à Toulon, le 25 juillet 1828, il annonce à son frère avoir découvert dans un papyrus appartenant à Sallier « les noms d'une quinzaine de nations vaincues, parmi lesquelles sont spécialement nommés les Ioniens, Iouni, Iauani (...) ${ }^{141}$ ». Il a donc trouvé un usage phonétique au signe du lièvre, qu'il ne précise pas encore, servant à composer des noms étrangers. Son dictionnaire ${ }^{142}$, publié après sa disparition, nous éclaire sur la valeur qu'il accorde alors à ce phonogramme pour composer des mots purement égyptiens: "Caractère phonétique, représentant une espèce de lièvre, et exprimant les diphtongues $\mathrm{OY}, \mathrm{OY} \mathrm{W}, \mathrm{OYO}(. .$.$) . » Il ne sait donc pas qu'il s'agit$ du bilitère $w n$.

Jusqu'à la fin de sa vie, Champollion lit «à la louche", de façon globale. Il ignore l'existence des bilitères, trilitères et quadrilitères, ainsi que l'antéposition honorifique. Il analyse les hiéroglyphes avant tout comme des idéogrammes dont la lecture peut être précisée par les signes de son «alphabet». De plus, il pense en copte, non seulement les mots mais aussi l'ensemble des énoncés, sans s'embarrasser des spécificités grammaticales des différentes époques qui correspondent à l'évolution normale d'une langue. De fait, il transcrit un nombre conséquent de mots avec des valeurs phonétiques fausses. Certaines comme «mammisi ${ }^{143}$ " (pour pr ms.t, litt. «maison de naissance ») sont encore employées par les égyptologues. Cependant, sa 
méthode improbable est efficace car la plupart de ses traductions sont justes. Ses lettres d'Égypte, sa grammaire ${ }^{144}$ et son dictionnaire ${ }^{145}$ publiés par son frère après sa mort en témoignent clairement.

\section{Conclusion}

\section{Comment et pourquoi Champollion a-t-il réussi?}

Comment Champollion a-t-il fait? Qu'est-ce qui a fait la différence? En fait, Champollion a osé désobéir aux préceptes de Barthélemy. Dans le système de lecture de Champollion, un même signe peut avoir plusieurs valeurs, qu'il exprime un son ou une idée. De plus, une même consonne peut être rendue par un nombre notable de signes différents. Ce nombre est d'autant plus important que Champollion prend pour des lettres alphabétiques certains bilitères, c'est-à-dire des signes qui expriment deux consonnes. De fait, il ne prononce jamais le nom de Barthélemy, ni dans la Lettre à Monsieur Dacier, ni dans son discours au Collège de France. Cela constitue un aveu car il y fait allusion: «Il y a longtemps que des savants du premier ordre avaient signalé ces encadrements elliptiques ou cartouches, comme constituant des noms propres ${ }^{146}$ ». Champollion est donc responsable de l'oubli injuste dans lequel est tombé Barthélemy. C'est comme si Einstein avait occulté Newton!

Comment Champollion a-t-il osé transgresser ? Il s'est intéressé à la théorie de Joseph de Guigne concernant l'origine égyptienne de l'écriture chinoise. Il a fini par conclure qu'elle n'était pas sensée. Ce faisant, il a étudié le chinois, avec un grand sérieux semble-t-il. Les échanges épistolaires entre les deux frères Champollion témoignent de l'intérêt de Jean-François pour ce système d'écriture depuis le début de sa quête ${ }^{147}$. Il entretient des rapports étroits avec le sinologue Abel Rémuzat ${ }^{148}$ (1788-1842) et il apprend même le chinois à l'École spéciale des langues orientales fondée en 1795. Le nombre d'évocations du chinois dans ses publications reflète l'importance de ce dernier dans sa démarche pour déchiffrer l'égyptien. Ainsi, par exemple, dans la Lettre à Monsieur Dacier, à propos des noms étrangers transcrits en démotique : «Les Chinois, qui se servent également d'une écriture idéographique, emploient aussi un procédé tout-à-fait semblable et créé pour le même motif ${ }^{149}$." Même s'il est conscient des différences entre les systèmes chinois et égyptien, Champollion constate que le précepte de Barthélemy concernant l'unicité de valeur des signes ne s'applique pas à l'écriture chinoise. Son esprit est alors débridé sur ce point, il peut envisager un système scriptural ouvert et complexe.

Limportance qu'a jouée l'analyse du système chinois d'écriture dans le déchiffrement de l'égyptien hiéroglyphique par Jean-François Champollion se reflète dans la préface du dictionnaire publié par son frère ${ }^{150}$.

Champollion le jeune examine ensuite, mais sommairement, quels peuvent être les rapports matériels de l'écriture hiératique égyptienne avec l'écriture actuelle des Chinois ; mais bientôt, il abandonne toute conjecture à former sur des analogies qui ne sont qu'apparentes, et qui lui semblent moins probables à mesure qu'il pénètre plus intimement dans les éléments du système hiératique égyptien ${ }^{151}$.

À la lecture de son introduction, on sent la gêne de Champollion-Figeac et ses efforts pour éviter les critiques à l'encontre de son frère. Il compare longuement les deux langues et les deux systèmes d'écriture, montrant que ces idiomes ne présentent aucun point commun, que les deux inventions sont indépendantes, l'égyptienne étant 
beaucoup plus ancienne, en dépréciant considérablement le chinois - d'une façon qui nous paraît caricaturale - afin de mettre en valeur le génie des Égyptiens. Il présente la pauvreté phonétique des mots chinois, l'extrême homophonie des signes chinois imposant la présence de clefs de lecture comparables aux déterminatifs de l'écriture égyptienne ${ }^{152}$, en hiéroglyphes, hiératique ou démotique.

Tel est l'avantage de l'écriture tachygraphique égyptienne sur l'écriture tachygraphique des Chinois: l'isolement absolu des signes fait le mérite de la première, et leur mélange tout le désavantage de la seconde ${ }^{153}$.

\section{Légende et réalité}

61 Par le décret du 15 mai 1826, Champollion devient conservateur chargé des collections égyptiennes du musée du Louvre.

Il y aura chaque année au musée des antiques du Louvre et durant la belle saison un cours public et gratuit d'archéologie où l'on exposera les différents systèmes d'écritures dont se servaient les Égyptiens et que le Sieur Champollion le jeune est chargé de ce cours ${ }^{154}$.

Ce premier projet avortera mais qui sait quelle aurait été l'histoire de cet enseignement au Louvre si Champollion avait vécu... En effet, il ne s'agit ni plus ni moins que des prémices de la création de l'École du Louvre. Toujours est-il qu'il faudra attendre 1882, lorsque l'idée sera reprise par Jules Ferry, pour qu'enfin le projet se concrétise. Il est notable que se sera encore une fois un projet porté par le désir d'enseigner l'archéologie et l'égyptologie, matière intimement liée à l'histoire de l'École des origines à nos jours. Deux ans après sa nomination au Louvre, Champollion voyage enfin en Égypte et peut traduire un nombre quasi illimité d'inscriptions qui couvrent les monuments pharaoniques. Il y écrit un courrier remarquable qui reflète son succès. En 1831, il devient professeur au Collège de France, comme en témoigne la statue de marbre qui nous accueille à l'entrée. À sa mort prématurée, en 1832, il est enterré au Père Lachaise. La grammaire égyptienne et le dictionnaire égyptien sont achevés et publiés par son frère.

La supposée compétition entre Young champion de l'Angleterre et Champollion celui de la France vient probablement du fait que celui-ci minimise le rôle des découvertes de Young dans son déchiffrement des hiéroglyphes. De son côté, Young reconnaît à Champollion la paternité du déchiffrement des hiéroglyphes, mais il désire que ce dernier reconnaisse à son tour s'être appuyé sur ses découvertes. En fait, les deux ont raison. Il est évident que Champollion n'est pas un extraterrestre représentant d'une génération spontanée, un génie révolutionnaire sans racines, qui aurait pu déchiffrer les hiéroglyphes n'importe où et n'importe quand. Barthélemy, Guigne, Sylvestre de Sacy, Åkerblad, Young et ceux qui les ont précédés ont posé les fondations sur lesquelles Champollion a construit son édifice. Cependant, sa réussite réside dans le fait qu'il soit le seul à oser désobéir aux préceptes de Barthélemy et à oser accepter la complexité du système hiéroglyphique : l'homophonie, l'homosémie, la polyphonie et la polysémie des signes, tout en étant le seul capable de comprendre ce qui était utilisable dans les Hiéroglyphica d'Horapollon. À ce titre, effectivement, il ne doit rien à personne; sa pensée est même opposée à la méthode scientifique en vigueur à son époque comme à la nôtre. Aujourd'hui, notre société ne lui laisserait aucune chance et les hiéroglyphes ne seraient pas déchiffrés. Heureusement pour l'égyptologie, il a vécu au $\mathrm{XIX}^{\mathrm{e}}$ siècle. Mais c'est aussi Champollion lui-même qui est à l'origine de la 
déformation patriotique de sa figure en présentant l'archéologie égyptienne comme « une sorte de propriété littéraire » de la France qui rétablit ses droits face à la victoire anglaise de $1801^{155}$. De plus, l'hagiographie a fait de lui un génie isolé, seul face aux hiéroglyphes. Pourtant, il n'était pas plus seul que Ramsès II face aux Hittites à la bataille de Qadech. Il était en contact avec les savants de son temps et continuellement guidé par son frère aîné. Champollion est donc un être bicéphale composé de deux frères géniaux : le cadet, Jean-François, et l'aîné, Jacques-Joseph ${ }^{156}$ qui avait discerné très tôt les capacités exceptionnelles de son jeune frère.

L'auteur tient à remercier particulièrement le personnel de la bibliothèque universitaire d'Heidelberg.

\section{NOTES}

1. Ammien Marcellin, Histoire de Rome, XVII, 4, 17-23.

2. Sur l'identification d'Hermapion, voir en dernier lieu Amin Benaissa, "Ammianus Marcellinus Res Gestae 17.4.17 and the Translator of the Obelisk in Rome's Circus Maximus », ZPE 186 (2013), p.114-118, qui propose, de façon assez convaincante, d'identifier cet Hermapion inconnu par ailleurs à Apion qui vit au $\mathrm{I}^{\mathrm{er}}$ siècle de notre ère.

3. Traduction Bérénice Lambrecht, «L'obélisque d'Hermapion (Ammien Marcellin, Res Gestae XVII, 4, 17-23) », Muséon 114 - 1-2 (2001), p. 53.

4. Idem, Ibidem, p. 54.

5. Idem, Ibidem.

6. Idem, Ibidem.

7. Idem, Ibidem.

8. Idem, Ibidem.

9. Idem, Ibidem, p. 55.

10. Grâce aux travaux de B. Lambrecht, nous savons aujourd'hui que c'est l'obélisque de la Piazza del Popolo inscrit aux noms de Séthi I ${ }^{\text {er }}$ et Ramsès II et que la traduction livrée par Ammien Marcellin n'est pas littérale. B. Lambrecht, op. cit. note 3, pp. 51-95.

11. Horapollon, Hiéroglyphica, Livre 1, entrée 26; Baudoin van de Walle et Joseph Vergote, «Traduction des Hieroglyphica d'Horapollon », CdE 35 (janvier 1943), pp. 64, 65. Pour un exemple plus complexe, voir Dominique Farout, « La voix du scarabée n'est pas à sens unique », Archéothéma 37 (novembre-décembre 2014), pp. 26-31.

12. Horapollon, Hiéroglyphica, livre 1, entrée 59 ; B. van de Walle et J. Vergote, op. cit. note 11, pp. 84, 85.

13. Horapollon, Hiéroglyphica, livre 1, entrée 61 ; B. van de Walle et J. Vergote, op. cit. note 11 , pp. $84-87$. 
14. Wb II, 89, 2. B. van de Walle et J. Vergote, op. cit. note 11, p. 85, proposent : « Meisi

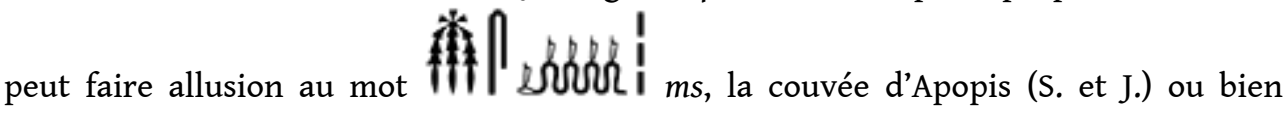
représenter le nom néo-égyptien du cartouche mnš (J.) ».

15. Horapollon, Hiéroglyphica, livre 2, entrée 76; B. van de Walle et J.Vergote, «Traduction des Hieroglyphica d'Horapollon », $C d E n^{\circ} 36$ (juillet 1943), pp. 226-227.

16. On retrouve cette histoire chez un certain nombre d'auteurs de l'Antiquité et de pères de l'Église. B. van de Walle et J. Vergote, op. cit. note 15, p. 227.

17. Jean Winand, «Les auteurs classiques et les écritures égyptiennes: quelques questions de terminologie ", dans Christian Cannuyer et al. (éd.), La langue dans tous ses états. Michel Malaise in honorem, Buxelles - Lièges - Louvain, 2005, pp.79-104, en particulier p. 92.

18. Horapollon, Hiéroglyphica, livre 2, entrée 113 ; B. van de Walle et J. Vergote, op. cit. note 15, pp. 236, 237.

19. Magdy Samy Zaky, Histoire des Coptes d'Égypte, Éditions de Paris, 2005.

20. Stéphane Pasquali, «Les hiéroglyphes égyptiens vus par les auteurs arabes du Moyen Âge ou l'aura du passé pharaonique ", dans cat. d'exp., Laure Bazin Rizzo, Annie Gasse, Frédéric Servajean (dir.), À l'école des scribes. Les écritures de l'Égypte ancienne, Silvana Editoriale, 2016, pp. 213-225.

21. Werner Vycichl, Dictionnaire étymologique de la langue copte, Louvain, Peeters, 1983, p. 176.

22. Voir Plutarque, Quaestiones convivales IX, 3, $738 \mathrm{E}$.

23. Sur cette question, voir en dernier lieu Didier Devauchelle, «L'alphabet des oiseaux (O dém. DelM 4-2)», dans Aidan M. Dodson, John J. Johnston et Wendy Monkhouse (éd.), A Good Scribe and an Exceedingly Wise Man. Studies in Honour of W. J. Tait, Londres, Golden House Publications, 2014, pp. 57-65.

24. Ben Haring, " Halaİam on an Ostracon of the Early New Kingdom? », JNES 74 (2015), pp. 189-196; D. Farout, "Écriture et pouvoir en Égypte et au Levant ", à paraître ; D. Farout, «La quête de l'histoire de l'alphabet », à paraître dans la revue NeHet.

25. Blaise de Vigenère, Traicté des chiffres, ov secretes manieres d'escrire, Paris, A. L'Angelier, 1586, Source gallica.bnf.fr.

26. Idem, Ibidem, fol. $54 \mathrm{r}^{\circ}$.

27. Idem, Ibidem, fol. $33 \mathrm{r}^{\circ}$.

28. Cette conception n'est pas propre à Vigenère, on la retrouve, par exemple, chez Étienne Guichard, L'harmonie etimologique des langues où se demonstre evidemment par plusieurs antiquitez curieusement recherchees que toutes les langues sont descenduës de l'hebraïque. Le tout disposé selon l'ordre alphabeticque avec deux tables l'une des mots grecs, l'autre des latins \& langues vulgaires, Paris, G. Le Noir, 1606 (2e éd. Victor Le Roy, 1618).

29. B. de Vigenère, op. cit. note 25 , fol. $162 \mathrm{v}^{\circ}$.

30. Idem, Ibidem, fol. $286 \mathrm{r}^{\mathrm{o}}-\mathrm{v}^{\mathrm{o}}$.

31. Idem, Ibidem, fol. $128 \mathrm{r}^{\mathrm{o}}$.

32. Idem, Ibidem, fol. $142 \mathrm{r}^{\mathrm{o}}-\mathrm{v}^{\mathrm{o}}$.

33. Plutarque, Isis et Osiris, traduction nouvelle avec Avant-propos, Prolégomènes et Notes par Mario Meunier, Paris, 1992, p. 48. Diodore de Sicile, I, 11, livre les mêmes explications. 
34. Jean-Jacques Barthélemy, « Réflexions sur quelques monumens phéniciens et sur les alphabets qui en résultent ", Mémoires de l'Académie des Belles Lettres t.XXX, 1758, pp. 405-427. D. Farout, « Le premier déchiffreur : l'Abbé Barthélemy », Égypte Afrique \& Orient 47 (septembre-novembre 2007), pp. 11-18.

35. Alan H. Gardiner, "The Egyptian Origin of the Semitic Alphabet », JEA 3 (1916), pp. 1-16, pl. I ; D. Farout, "Les hiéroglyphes et la naissance de l'alphabet ", Égypte Afrique \& Orient 46 (juin 2007), pp. 37-48.

36. B. de Vigenère, op. cit. note 25 , fol. $296 \mathrm{r}^{\circ}$.

37. Idem, Ibidem, fol. $307 \mathrm{v}^{\circ}-308 \mathrm{r}^{\circ}$.

38. Idem, Ibidem, fol. $296 \mathrm{v}^{\circ}$.

39. Idem, Ibidem, fol. $297 \mathrm{r}^{\mathrm{o}}-\mathrm{v}^{\mathrm{o}}$; voir aussi fol. $298 \mathrm{r}^{\mathrm{o}}-\mathrm{v}^{\mathrm{o}}$.

40. Idem, Ibidem, fol. $314 \mathrm{v}^{\mathrm{o}}-315 \mathrm{r}^{\mathrm{o}}$.

41. Werner Vycichl, Dictionnaire étymologique de la langue copte, Louvain, Peeters, 1983, p. 5.

42. B. de Vigenère, op. cit. note 25 , fol. $10 \mathrm{v}^{\mathrm{o}}$.

43. Idem, Ibidem, fol. $317 \mathrm{v}^{\mathrm{o}}-318 \mathrm{r}^{\mathrm{o}}$.

44. Idem, Ibidem, fol. $323 \mathrm{r}^{\mathrm{o}}-\mathrm{v}^{\mathrm{o}}$.

45. Idem, Ibidem, fol. $325 \mathrm{r}^{\circ}$.

46. Idem, Ibidem, fol. $325 \mathrm{v}^{\circ}$.

47. Werner Vycichl, op. cit. note 41, p. 81.

48. Paula Findlen (éd.), Athanasius Kircher. The Last Man Who Knew Everything, New York et Londres, Routledge, 2004.

49. Athanasius Kircher, Obelisci Aegyptiaci nuper inter isaei romani rudera effossi interpretatio hieroglyphica, Rome, Varesi, 1666, p. 53.

50. Grès silicifié, H. : $92 \mathrm{~cm}$; L. : $55 \mathrm{~cm}$; Pr. : $30 \mathrm{~cm}$; Idem, Ibidem, face à la p. 136 ; Élisabeth Delange, Catalogue des statues égyptiennes du Moyen Empire, 2060-1560 avant J.-C., Paris, Éd. de la Réunion des musées nationaux, 1987, pp. 81-83.

51. Ou : " le grand des conducteurs des artisans ». Pour une bibliographie concernant ce titre, voir Bernard Mathieu, "Réflexion sur le "fragment Daressy" et ses hommes illustres ", dans Christiane Zivie-Coche et Ivan Guermeur (dir.) "Parcourir l'éternité»: Hommages à Jean Yoyotte, Turnhout, Brepols, 2012, p. 828, note 36.

52. À mon avis, ce Nebipou de la fin de la XII ${ }^{\mathrm{e}}$ dynastie doit être distingué du grand prêtre de Ptah Nébipounyrê représenté sur le fragment Daressy, qui vit à la XIII ${ }^{\mathrm{e}}$ dynastie, contra B. Mathieu, op. cit. note 51, p. 831. En dernier lieu, la partie inférieure d'une statue assise en tailleur asymétrique en calcaire au nom de ce personnage a été découverte à Hazor, en juillet 2016 lors de la campagne de fouilles de la «Selz Fondation Hazor Excavations in Memory of Yigael Yadin " sous la direction d'Amnon Ben-Tor. http://new.huji.ac.il/en/article/31994 «Historic Find at Tel-Hazor: A Statue of an Egyptian Official 25/07/2016 » [consulté le 18/08/2016].

53. Madeleine V. David, Le débat sur les écritures et l'hiéroglyphe aux XVII et XVIII siècles, Paris, S.E.V.P.E.N, 1965.

54. Il publie en 1641 Mercury or the secret and swift messenger au titre évocateur, et en 1668, An essay towards a real character and a philosophical language. 
55. Réédité à Paris en 1977, aux éditions Aubier Flammarion, collection Palimpseste.

56. Cité par M. V. David, «Communication en marge du mémoire de l'abbé Barthélemy sur les inscriptions phéniciennes (1758) », CRAIBL, 105 année, N. 1 (1961), p. 31.

57. James Dawkins et Robert Wood, The Ruins of Palmyra otherwise Tadmor in the Desart, Londres, Robert Wood, 1753.

58. Jean-Jacques Barthélemy, «Réflexions sur l'alphabet et sur la langue dont on se servoit autrefois à Palmyre ", Mémoires de l'Académie des Belles Lettres, t. XXVI, 1754, pp. 577-597, pl. I-III ; CRAIBL, source Gallica.bnf.fr ; Dominique Farout, «Le premier déchiffreur: l'Abbé Barthélemy", Égypte Afrique \& Orient 47 (septembre-novembre 2007), pp. 12-15.

59. "Réflexions sur quelques monumens phéniciens et sur les alphabets qui en résultent ", Mémoires de l'Académie des Belles Lettres, t. XXX, 1758, pp. 405-427 ; D. Farout, "Le premier déchiffreur: l'Abbé Barthélemy ", Égypte Afrique \& Orient 47 (septembrenovembre 2007), p. 15.

60. Sur les noms des dédicants formés sur Osiris, voir Corinne Bonnet, "Stratégies d'intégration des cultes isiaques et du culte des Lagides dans la région de Tyr à l'époque hellénistique », dans Gaëlle Tallet et Christiane Zivie-Coche (éd.), Le myrte \& la rose. Mélanges offerts à Françoise Dunand par ses élèves, collègues et amis, CENIM 9 (2014), p. 39.

61. André Dupont-Sommer, "Jean-Jacques Barthélemy et l'ancienne Académie des Inscriptions et Belles-Lettres », CRAIBL, vol. 115, 4 (1971), pp. 716, 717.

62. J.-J. Barthélemy, «Réflexions générales sur les rapports des langues égyptienne, phénicienne et grecque ", Mémoires de l'Académie, t. XXXII, 1763, p. 212.

63. Joseph de Guignes, Mémoire dans lequel on prouve que les Chinois sont une colonie égyptienne. Lu dans l'Assemblée publique de l'Académie Royale des inscriptions \& belles-lettres, le 14 novembre 1758, Paris, De Saint \& Saillant, 1759. Édition en format texte par Pierre Palpant, www.chineancienne.fr, novembre 2013.

64. Il n'est pas le premier à l'énoncer: Pierre-Daniel Huet, Histoire du commerce et de la navigation des Anciens, Paris, A.-N. Constelier, 1716 ; Hermine Hartleben, Champollion sein Leben und sein Werk, Berlin, Weidmannsche Buchhandlung, 1906, pp. 356-358.

65. Hermine Hartleben, Champollion, Paris, Pygmalion, 1983, p. 199.

66. J. de Guignes, op. cit. note 63, p. 9 (Extrait de la lettre du père Parrenin).

67. J. de Guignes, op. cit. note 63, pp. 17, 27-28 ; Alan H. Gardiner, « The Egyptian Origin of the Semitic Alphabet », JEA 3 (1916), pp. 1-16

68. Sur l'imprimerie dans le monde arabo-musulman, voir G. Oman, «Mațba'a», dans Encyclopédie de l'Islam, fasc. 111, pp. 784-787.

69. Cependant, l'imprimerie de Constantinople fondée par Zaïd Aga et Ibrahim Müteferrika a été rapidement fermée pour des raisons politico-religieuses, en fait sous la pression des copistes.

70. $\mathrm{H} 1,12-1,14 \mathrm{~m} ; 1$ 72-75,7 cm ; E 27-28,4 cm ; 760-762 kg.

71. Silvestre de Sacy, Lettre à M. Chaptal, Ministre de l'Intérieur, au sujet de l'Inscription égyptienne du Monument trouvé à Rosette, Paris, Imprimerie de la République, 1802, t. I, p. 246. 
72. Johan David Åkerblad, Lettre sur l'inscription égyptienne de Rosette, Adressée au Citoyen Sylvestre de Sacy, Professeur de langue Arabe à l'école spéciale des langues Orientales vivantes, etc., Paris, Imprimerie de la République, 1802.

73. Sur ce point, voir en particulier Alain Faure, Champollion, le savant déchiffré, Paris, Fayard, 2004.

74. H. Hartleben, Champollion, op. cit. note 65, p. 61.

75. A. Faure, Champollion, le savant déchiffré, op. cit. note 73, p. 52.

76. Marc Gabolde, « Une lettre inédite de Jean-François Champollion à la bibliothèque de l'académie de Lyon », Bulletin du Cercle Lyonnais d'Égyptologie Victor Loret $n^{\circ} 4$ (1990), p. 7 : « conservée à la bibliothèque de l'Académie des Sciences, Belles-lettres \& Arts de Lyon, dans le Recueil 287 dont elle est la $13^{\mathrm{e}}$ pièce ».

77. Idem, Ibidem, p. 10.

78. Idem, Ibidem, pp. 9, 32.

79. Idem, Ibidem, pp. 9, 33.

80. Idem, Ibidem, pp. 11, 34 .

81. Idem, Ibidem, p. 10.

82. Jean Leclant, «Champollion, la pierre de Rosette et le déchiffrement des hiéroglyphes », CRAIBL, $116^{\mathrm{e}}$ année, $\mathrm{n}^{\circ} 3$ (1972), p. 562.

83. Champollion, Lettre à M. Dacier, secrétaire perpétuel de l'Académie Royale des Inscriptions et Belles-Lettres, relative à l'alphabet des hiéroglyphes phonétiques employés par les Égyptiens pour inscrire sur leurs monuments les titres, les noms et les surnoms des souverains grecs et romains, Paris, Firmin Didot, 1822.

84. Idem, Ibidem, p. 3.

85. Idem, Ibidem, pp. 1-2.

86. Idem, Ibidem, p. 5 .

87. Idem, Ibidem, p. 5

88. Il précise que l'écriture phonétique égyptienne leur est comparable dans la Lettre à M. Dacier, p. 34.

89. Jean-Antoine Letronne, Éclaircissements sur une inscription grecque, contenant une pétition des prêtres d'Isis, dans l'île de Philae, à Ptolémée Évergète second, copiée à Philøe, par M. Cailliaud, en octobre 1816, Paris, Académie royale des Inscriptions et Belles-Lettres, 1822.

90. Champollion, Lettre à M. Dacier, op. cit. note 83, pp. 6, 19-20.

91. Idem, Ibidem, pl. $\mathrm{I}^{\mathrm{e}}, 21$. «Signe idéographique du genre féminin. »

92. Idem, Ibidem, pp. 6-7.

93. Idem, Ibidem, pp. 8-9.

94. Idem, Ibidem, pp. 20, 26, 28, 29.

95. Idem, Ibidem, pp. 20, 31.

96. Idem, Ibidem, p. 20.

97. Idem, Ibidem, pp. 10, 14, 15, 16, 19

98. Idem, Ibidem, p. 27.

99. Idem, Ibidem, pp. 17-18.

100. Idem, Ibidem, pp. 17, 20, 21, 25, 26, 33. 
101. Idem, Ibidem, p. 17

102. Idem, Ibidem, pp. 29, 35.

103. Idem, Ibidem, p. 31.

104. Idem, Ibidem, pp. 17, 20.

105. Idem, Ibidem, pp. 17-20.

106. Idem, Ibidem, p. 19.

107. Idem, Ibidem, pp. 19, 20.

108. Idem, Ibidem, pp. 21-23.

109. Idem, Ibidem, p. 23.

110. Idem, Ibidem, p. 18.

111. Idem, Ibidem, p. 19.

112. Idem, Ibidem, p. 19.

113. Idem, Ibidem, p. 24.

114. Idem, Ibidem, pp. 26-29, 31, 32.

115. Idem, Ibidem, p. 24.

116. Idem, Ibidem, p. 25.

117. Idem, Ibidem, p. 27.

118. Idem, Ibidem, p. 28.

119. Idem, Ibidem, pp. 28-29.

120. Idem, Ibidem, p. 29.

121. Idem, Ibidem, pp. 29-31

122. Idem, Ibidem, p. 31

123. Idem, Ibidem, p. 32.

124. Idem, Ibidem, p. 32-33.

125. Idem, Ibidem, p. 2.

126. Idem, Ibidem, p. 40-42.

127. Pierre Pinon, «L'Orient de Jean Nicolas Huyot: le voyage en Asie-Mineure, en Égypte et en Grèce (1817-1821) ", Revue du monde musulman et de la Méditerranée 73-74 (1994), pp. 35-55.

128. J.-F. Champollion, Lettre à M. Dacier, op. cit. note 83, p. 44.

129. J.-F. Champollion, Précis du système hiéroglyphique des Anciens Égyptiens, Paris, L'Imprimerie royale, 1824, pp. 1-2.

130. Idem, Ibidem, pp. 11-12.

131. Lettres de Champollion le jeune recueillies et annotées par H. Hartleben, Tome deuxième, Lettres et journaux écrits pendant le voyage d'Égypte, Paris, Ernest Leroux, 1909, p. 94.

132. Idem, Ibidem, p. 95.

133. Idem, Ibidem, p. 94.

134. Idem, Ibidem, p. 100.

135. Idem, Ibidem, p. 261.

136. Idem, Ibidem, p. 94. 
137. Idem, Ibidem, p. 96.

138. Idem, Ibidem, p. 95.

139. Idem, Ibidem, p. 105.

140. Lettres de Champollion le jeune recueillies et annotées par H. Hartleben, Tome premier. Lettres écrites d'Italie, Paris, Ernest Leroux, 1909, « Réponse de M. Champollion le jeune à la lettre de M. le Baron Guillaume de Humboldt », pp. 144-166 ; le passage concernant le lièvre se trouve pp. 162-163.

141. Lettres de Champollion le jeune recueillies et annotées par H. Hartleben, Tome deuxième, Lettres et journaux écrits pendant le voyage d'Égypte, Paris, Ernest Leroux, 1909, p. 11.

142. J.- J. Champollion-Figeac, Dictionnaire égyptien en écriture hiéroglyphique par J. F. Champollion le jeune; publié d'après les manuscrits autographes, et sous les auspices de M. Villemain ministre de l'instruction publique, Paris, Firmin Didot, 1841-1843, nº 106, p. 125.

143. Lettres de Champollion le jeune recueillies et annotées par H. Hartleben, Tome deuxième, op. cit. note 131, p. 258 « lieu d'accouchement».

144. Grammaire égyptienne, ou Principes généraux de l'écriture sacrée égyptienne appliquée à la représentation de la langue parlée, Par Champollion le jeune; publiée sur le manuscrit autographe, par ordre de M. Guizot, Ministre de l'Instruction publique, Paris, 1836 ; réédition Institut d'Orient, Paris, 1984 ; réédition Jean de Bonnot, Paris, 1994.

145. J.- J. Champollion-Figeac, Dictionnaire égyptien en écriture hiéroglyphique par J. F. Champollion le jeune, op. cit. note 142.

146. J.-F. Champollion, Précis du système hiéroglyphique des anciens Égyptiens, op.cit. note 129 , p. 22.

147. Sur l'intérêt de Champollion pour le chinois, voir H. Hartleben, Champollion sein Leben und sein Werk, op. cit. note 64, pp. 40, 48, 80, 103, 124, 151, 207, 300, 313, 346, 366, 370, 375, 377, 378, 379, 390, 394, 402, 436, 439.

148. A. Faure, Champollion, le savant déchiffré, op. cit. note 73, pp. 118, 157, 417, 466, 558.

149. J.-F. Champollion, Lettre à M. Dacier, op. cit. note 83, p. 4, voir aussi p. 11.

150. J.- J. Champollion-Figeac, Dictionnaire égyptien en écriture hiéroglyphique par J. F. Champollion le jeune, op. cit. note 142, préface de l'éditeur pp. XV-XXVII.

151. Idem, Ibidem, préface de l'éditeur, p. VI.

152. Idem, Ibidem, préface de l'éditeur, pp. XXI-XXIV.

153. Idem, Ibidem, préface de l'éditeur, p. XXI.

154. J.-F. Champollion, Notice descriptive des monuments égyptiens du musée Charles $X$, édition établie par Sylvie Guichard, Paris, Éditions Khéops et Louvre éditions, 2013, p. 26.

155. Éric Gady, «Les égyptologues français au $\mathrm{XIX}^{\mathrm{e}}$ siècle: quelques savant très influents ", Revue d'histoire du XIX siècle 32 (2006), p. 46.

156. Charles-Olivier Carbonell, L'Autre Champollion: Jacques-Joseph Champollion-Figeac (1778-1867), Toulouse, Presses de l'Institut d'études politiques de Toulouse, 1984. 


\section{RÉSUMÉS}

Le déchiffrement de l'égyptien hiéroglyphique n'est pas l'œuvre miraculeuse d'un génie isolé, mais l'aboutissement d'une quête qui a duré plusieurs siècles, impliquant de nombreux savants. Sa cause remonte à la redécouverte du passé antique à la Renaissance. Chacun des acteurs qui se sont succédés a ajouté à l'édifice la pierre qui correspondait au savoir de son temps. De plus, des traditions vivaces dans l'Égypte du Moyen Âge sont à l'origine de certaines notions fondamentales, comme le lien entre le copte et l'écriture pharaonique. Enfin, la découverte du système hiéroglyphique est l'œuvre des deux frères Champollion, l'aîné guidant le jeune qui n'a pas tout déchiffré en un instant, un jour de 1822.

Deciphering Egyptian hieroglyphs is not the miraculous achievement of one isolated genius, but rather the result of a long quest which covered several centuries and involved many scholars. Its origin dates back to the rediscovery of Antiquity during the Renaissance. Each one of these figures successively contributed to the project using the knowledge at their disposal at the time. In addition to this, some significant traditions from the Egyptian Middle Ages are at the root of fundamental concepts such as the link between Coptic and Pharaonic writing. Lastly, the discovery of the hieroglyphic system is the work of Champollion brothers, the elder guiding the younger: the latter did not simply decipher it all in an instant, one fine day in 1822.

\section{INDEX}

Mots-clés : Hermapion, Horapollon, Vigenère, Kircher, Warburton, Barthélemy, pierre de Rosette, Åkerblad, Young, Champollion

Keywords : Hermapion, Horapollon, Vigenère, Kircher, Warburton, Barthélemy, Rosetta Stone, Åkerblad, Young, Champollion

\section{AUTEUR}

\section{DOMINIQUE FAROUT}

Dominique Farout enseigne la langue et la civilisation égyptienne à l'Institut Khéops depuis 1987, à l'École du Louvre depuis 1991, à l'École normale supérieure rue d'Ulm depuis 1996 et à l'Institut catholique de Paris depuis 2009. Il est également chercheur associé, PLH-CRATA, à l'université Jean-Jaurès de Toulouse.

Dominique Farout has been teaching Egyptian language and civilisation at the Institut Kheops since 1987, at the École du Louvre since 1991, at the École normale supérieure since 1996 and at the Institut catholique of Paris since 2009. He is also a research fellow and member of the PLHCRATA research team at the Jean-Jaurès University of Toulouse. 